\title{
SCR Device With Double-Triggered Technique for On-Chip ESD Protection in Sub-Quarter-Micron Silicided CMOS Processes
}

Ming-Dou Ker, Senior Member, IEEE, and Kuo-Chun Hsu, Student Member, IEEE

Nanoelectronics and Gigascale Systems Laboratory

Institute of Electronics, National Chiao-Tung University

1001 Ta-Hsueh Road, Hsinchu, Taiwan

Tel: (+886)-3-5131573, Fax: (+886)-3-5715412, E-mail: mdker@ieee.org

\begin{abstract}
Turn-on efficiency is the main concern for silicon controller rectifier (SCR) devices used as on-chip electrostatic discharge (ESD) protection circuit, especially in deep sub-quarter-micron CMOS processes with much thinner gate oxide. A novel double-triggered technique is proposed to speed up the turn-on time of SCR devices for using in on-chip ESD protection circuit to effectively protect the much thinner gate oxide in sub-quarter-micron CMOS processes. From the experimental results, the switching voltage and turn-on time of such double-triggered SCR (DT_SCR) device has been confirmed to be significantly reduced by this double-triggered technique.
\end{abstract}

Index Terms - Double-triggered technique, electrostatic discharge (ESD), ESD protection circuit, silicon controlled rectifier (SCR).

* This work was supported by the National Science Council, Taiwan under contract of NSC 91-2215-E-009-077.

** Copyright (c) 2003 IEEE. Personal use of this material is permitted. However, permission to use this material for any other purposes must be obtained from the IEEE by sending a request to pubs-permissions@ieee.org. 


\section{INTRODUCTION}

With the process evolutions, the gate oxide thickness has been scaled down to increase circuit operating speed under lower voltage supply. For oxide reliability concerns, the normal operating voltage has been limited to some voltage level for each CMOS technology. But, the electrostatic discharge (ESD) event often has a much higher overstress voltage, which can burn out the junction or rupture the gate oxide. Therefore, in order to effectively protect the thinner gate oxide from ESD stresses, on-chip ESD protection circuit must have lower clamping voltage and faster turn-on speed. Among the various ESD protection devices, silicon controlled rectifier (SCR) has the lowest holding voltage ( $\mathrm{V}_{\text {hold }}$, about $\sim 1 \mathrm{~V}$ in general CMOS processes). SCR is composed of parasitic NPN and PNP transistors in CMOS process, and its turn-on mechanism is essentially a current triggering event. With the best area-efficient ESD robustness, SCR had been used as on-chip ESD protection for a long time [1], [2]. But, SCR has some drawbacks in CMOS IC applications, such as higher switching voltage (Vt1) and latchup issues [3]-[5]. Some reports had presented the solutions to overcome these issues [6]-[8].

In this paper, the novel double-triggered SCR (DT_SCR) device is proposed and verified in a $0.25-\mu \mathrm{m}$ salicided CMOS process. The purpose of double-triggered technique is to reduce the Vt1 and turn-on time of SCR device. The DT_SCR device is designed to be triggered on without involving junction avalanche breakdown mechanism Therefore, the much thinner gate oxide in sub-quarter-micron CMOS processes can be effectively protected by such DT_SCR device. With a suitable ESD-detection circuit, such DT_SCR device is designed to be kept off during the normal circuit operating conditions, and to be quickly triggered on during the ESD-zapping conditions.

\section{Double-Triggered SCR DeVICE}

\section{A. Device Structure}

The proposed double-triggered SCR (DT_SCR) device is shown in Fig. 1. The ESD current path in the DT_SCR device is indicated by the dashed lines shown in Fig. 1. As comparing to the traditional lateral SCR device structure [1], extra $\mathrm{P}+$ and $\mathrm{N}+$ diffusions are inserted into the P-substrate and N-well of the DT_SCR device structure, respectively. The inserted P+ and N+

diffusions are connected out as the p-trigger and n-trigger nodes of the DT_SCR device. When a 
trigger current is applied into p-trigger node, the NPN bipolar transistor in SCR structure is active, and the collector current of NPN is generated to bias the PNP bipolar transistor. When PNP transistor is turned on, the collector current of PNP is also generated to further bias NPN transistor. The positive feedback regeneration mechanism [9] of latchup is initiated by the substrate-triggered current in SCR structure instead of avalanche breakdown mechanism, so the DT_SCR will be triggered into its latching state. When a trigger current is drawn out from the n-trigger node, the DT_SCR will be also triggered on into its latching state through the positive feedback regeneration mechanism In this work, two trigger currents can be synchronously applied to trigger on the DT_SCR device.

\section{B. Device Characteristics}

The fully-silicided DT_SCR device in Fig. 1 has been fabricated in a $0.25-\mu \mathrm{m}$ salicided CMOS process. The layout top view of the DT_SCR device is shown in Fig. 2. The active area of the DT_SCR device is $20 \mu \mathrm{m} \times 20 \mu \mathrm{m}$. Fig. 3(a) shows the measured DC I-V curves of DT_SCR, which is measured with different substrate-triggered currents into the p-trigger node of DT_SCR but no N-well triggered current. The measurement setup is shown as the inset in Fig. 3(a). The Vt1 of such DT_SCR is reduced with the increase of the substrate-triggered current. When the triggered current at the p-trigger node is increased from 0 to $6 \mathrm{~mA}$, the Vt1 of DT_SCR is reduced from $\sim 22$ to $\sim 7 \mathrm{~V}$. If the triggered current is continually increased, the Vt1 will be nearly reduced to its holding voltage. Moreover, in Fig. 3(b), the double-triggered solution is used to further reduce the $\mathrm{Vtl}$ to a relatively lower voltage level. Based on the similar measurement of Fig. 3(a), an extra $\mathrm{N}$-well current of $2 \mathrm{~mA}$ is drawn out from the n-trigger node of DT_SCR, and the measured FV curves under different substrate-triggered currents into the p-trigger node are shown in Fig. 3(b). The double-triggered measurement setup is also indicated in the inset of Fig. 3(b). The Vt1 of DT_SCR under the substrate-triggered current of $6 \mathrm{~mA}$ is further reduced from $\sim 7$ to only $\sim 2 \mathrm{~V}$, when the $\mathrm{N}$-well triggered current is increased from 0 to -2 $\mathrm{mA}$. The negative sign on the current in this paper is used to represent the current flowing out from the node. The dependence of switching voltage of DT_SCR device on the substrate-triggered current under different $\mathrm{N}$-well triggered currents is depicted in Fig. 3(c). The Vt1 of DT_SCR device can be reduced to nearly the holding voltage $(\sim 1.5 \mathrm{~V})$ more efficiently, when both the substrate-triggered and N-well triggered currents are applied to the DT_SCR 
device. These results have proven that the Vt1 of DT_SCR device can be significantly reduced by the proposed double-triggered technique.

The measured DC I-V curves of DT_SCR under a substrate-triggered current of $2 \mathrm{~mA}$ into the p-trigger node and different $\mathrm{N}$-well triggered currents out from the $\mathrm{n}$-trigger node are shown in Fig. 4(a). The measurement setup is also illustrated as the inset in Fig. 4(a). When the N-well triggered current is increased from 0 to $-3 \mathrm{~mA}$, the Vt1 of DT_SCR under the substrate-triggered current of $2 \mathrm{~mA}$ is significantly reduced from $\sim 15$ to only $\sim 1.5 \mathrm{~V}$, which is near to its holding voltage. The dependence of Vt1 of the DT_SCR on the N-well triggered current under different substrate-triggered currents is shown in Fig. 4(b). The Vt1 of DT_SCR under the N-well triggered current of $-3 \mathrm{~mA}$ is further reduced from $\sim 21$ to $\sim 1.5 \mathrm{~V}$, when the substrate-triggered current is increased from 0 to $2 \mathrm{~mA}$. The characteristics of DT_SCR in Fig. 4(b) are similar to that in Fig. 3(c). In the p-type substrate, because the current gain of NPN transistor is higher than that of PNP transistor, the substrate-triggered current used to trigger the NPN transistor in the DT_SCR device seems to have more significant effect, than N-well triggered current used to trigger the PNP transistor in the DT_SCR device, to reduce Vt1 of the DT_SCR and to quickly trigger on DT_SCR [10]. The ESD protection device with lower switching voltage can be turned on more quickly to protect the internal circuits from ESD damage.

Another issue of using SCR device as the ESD protection device is the transient-induced latchup concern, when CMOS IC is operating under normal circuit operations. The total holding voltage of the ESD protection circuit with SCR devices must be designed greater than the maximum voltage level of VDD during the normal circuit operating conditions to avoid the latchup issue. This can be achieved by stacking the DT_SCR devices in the ESD protection circuits. Fig. 5 shows the dependence of the total holding voltage of stacked DT_SCR devices on the temperature under different numbers of stacked DT_SCR devices. The measurement setup to measure the FV curves of stacked DT_SCR devices is depicted in Fig. 5(a). The FV curves of two (three) DT_SCR devices in stacked configuration, which is marked as 2DT_SCR (3DT_SCR), under different temperatures are measured in Fig. 5(b) (Fig. 5(c)). The insets in Figs. 5(b) and 5(c) are the enlarged views around the holding points. The total holding voltage has some degradation, when the temperature is increased, because the current gains (ß) of the parasitic bipolar transistors in the SCR device are increased with the increase of temperature. The holding voltages of 1DT_SCR, for example, are 1.4, 1.24, and $1.18 \mathrm{~V}$ under the temperatures of 
25,75 , and $125{ }^{\circ} \mathrm{C}$, respectively. The total holding voltage, however, can be still raised up by increasing the number of the stacked DT_SCR devices. The holding voltages of 1DT_SCR, 2DT_SCR, and 3DT_SCR at the temperature of $125^{\circ} \mathrm{C}$ are $1.18,2.5$, and $3.9 \mathrm{~V}$, respectively. The dependence of holding voltage on temperature in the stacked DT_SCR devices is compared in Fig. 5(d). Although the DT_SCR devices in stacked configurations have the increased total switching voltage, such stacked DT_SCR devices can be still quickly triggered on to provide effective ESD protection, when the double-triggered technique is synchronously applied to all stacked DT_SCR devices.

\section{Turn-on Speed}

From the measured DC I-V curves of DT_SCR, it has been verified that the Vt1 of DT_SCR can be significantly reduced by the substrate and $\mathrm{N}$-well triggered currents. The turn-on time of DT_SCR, which is defined as the time for DT_SCR entering into its latching state, will be verified in this section. Fig. 6(a) shows the measurement setup to find the turn-on time of DT_SCR devices with double-triggered technique. The measured results in time domain for DT_SCR are shown in Figs. 6(b) to 6(h), where the V_anode, V_p-trigger, and V_n-trigger are the voltage waveforms on the anode, p-trigger, and n-trigger nodes of the DT_SCR shown in Fig. 6(a). The anode of the DT_SCR device is biased at $5 \mathrm{~V}$ through the resistance of $10 \Omega$, which is used to limit the sudden large transient current from power supply, when the DT_SCR is turned on.

The positive and negative voltage pulses with a fixed rise time (or fall time) of $10 \mathrm{~ns}$, which are generated from pulse generators, are synchronously applied to the p-trigger and n-trigger nodes. The original voltage pulses generated synchronously from pulse generators are shown in Fig. 6(b). The pulse height and pulse width are changed in the experimental measurement to verify the required turn-on time of DT_SCR. However, in order to avoid the loading effect of oscilloscope interfering the accuracy of measured waveform, only the V_p-trigger will be monitored. When a 0 -to-1.5 $\mathrm{V}$ positive voltage pulse with a pulse width of $100 \mathrm{~ns}$ is applied into the p-trigger node of DT_SCR and the n-trigger node is floating, the voltage waveform at the V_anode of the DT_SCR which is triggered into latching state is shown in Fig. 6(c). After the triggering of $1.5-\mathrm{V}$ voltage pulse at the p-trigger node, the $\mathrm{V}$ _anode is latched at a low voltage level of $\sim 2.5 \mathrm{~V}$ and the $\mathrm{V} \_$p-trigger is kept at a voltage level of $0.8 \mathrm{~V}$. If the pulse width of $1.5-\mathrm{V}$ 
pulse at the p-trigger node is reduced to $30 \mathrm{~ns}$, the DT_SCR device can not be triggered on by this $1.5-\mathrm{V}$ voltage pulse. So, the V_anode is still kept at the same voltage level of $5 \mathrm{~V}$ as shown in Fig. 6(d). However, based on the same condition of Fig. 6(d), the DT_SCR device can be triggered into latching state if an additional 5-to- $0 \mathrm{~V}$ negative voltage pulse with pulse width of $30 \mathrm{~ns}$ is synchronously applied to the n-trigger node of DT_SCR device, as that shown in Fig. 6(e). So, the required pulse width for DT_SCR triggering into latching state can be shortened if both positive and negative voltage pulses are synchronously applied to the $\mathrm{p}$-trigger and n-trigger nodes.

The turn-on time for DT_SCR into its latching state is observed by the close-up view of the V_anode voltage waveform at the falling edge. The close-up views of the V_anode at the falling edge, while the DT_SCR is synchronously triggered by the positive voltage pulse of $1.5 \mathrm{~V}$ at p-trigger node and the negative voltage pulse of floating, 5-to-2 V, and 5-to-0 V at n-trigger node, are compared in Figs. 6(f), 6(g), and 6(h), respectively. The pulse widths of the positive and negative voltage pulses in the measurements of Figs. 6(f)-6(h) are 200 ns. The turn-on time of DT_SCR is $37.6 \mathrm{~ns}$ in Fig. 6(f) if only a 1.5-V positive voltage pulse is applied into the p-trigger node. Hence, the DT_SCR can not be triggered on, if only a $1.5-\mathrm{V}$ voltage pulse with a pulse width smaller than $37.6 \mathrm{~ns}$ is applied into the p-trigger node alone, which has been verified in Fig. 6(d). Moreover, from Figs. 6(f) to 6(h), under the positive voltage pulse of $1.5 \mathrm{~V}$ at p-trigger node, the turn-on time can be reduced from 37.6 to $11.8 \mathrm{~ns}$, while the absolute pulse height of negative voltage pulse applied to the n-trigger node is increased from 0 to $5 \mathrm{~V}$. These results infer that the turn-on speed of DT_SCR device can be indeed speeded up by the proposed double-triggered technique. The dependence of turn-on time of DT_SCR on the N-well bias under different substrate bias conditions with a fixed rise time of 10ns is summarized in Fig. 7. The turn-on time of DT_SCR can be shortened, when the substrate or / and N-well bias voltages are increased. In addition, the dependence of turn-on time of DT_SCR on rise time of voltage pulse under different substrate bias conditions is also measured and shown in Fig. 8. With the reduction of rise time of applied voltage pulse, the turn-on time of DT_SCR can be also shortened to trace the rise time of voltage pulse at the p-trigger node if the enough pulse voltage is applied to trigger nodes of DT_SCR. For enhance the turn-on speed of DT_SCR, both the pulse height and rise time must be well designed to trigger on the DT_SCR device more efficiently. 


\section{ApPlications For On-Chip ESD Protection}

\section{A. ESD Protection Circuit for the Input/Output Pad}

Based on above measured results, the ESD protection design for I/O pad, realized with the stacked double-triggered SCR devices, is shown in Fig. 9. Two stacked DT_SCR devices are used to avoid the latchup issue during normal circuit operating conditions for $2.5-\mathrm{V}$ circuit applications. The RC-delay circuit technique is used to distinguish the ESD-zapping conditions or the normal circuit operating conditions.

In Fig. 9, the p-trigger (n-trigger) nodes of the two stacked DT_SCR devices between I/O pad and VSS pin are connected to the drain (source) of PMOS Mp1 (Mp2). The p-trigger (n-trigger) nodes of the two stacked DT_SCR devices between I/O pad and VDD pin are connected to the source (drain) of NMOS Mn1 (Mn2). The gates of the PMOS Mp1 and Mp2 (NMOS Mn1 and $\mathrm{Mn} 2$ ) are connected to VDD (VSS) through the resistor R1 (R2), which is better realized by the $\mathrm{N}+$ diffusion resistor for the concern of antenna effect [11]. The resistors R1 and R2 can be shared by each I/O pad to save the layout area in the CMOS IC. A capacitor C1 (C2) is placed between the gates of PMOS (NMOS) and VSS (VDD). These capacitors can be formed by the parasitic capacitors at the gates of the PMOS (Mp1 and Mp2) or NMOS (Mn1 and Mn2). The blocking diodes, $\mathrm{Db}$, are used to block the current flowing through the metals connected among the trigger nodes of the stacked DT_SCR devices. Without the blocking diodes, the larger ESD current will flow out from the first p-trigger / n-trigger node of DT_SCR into metal connection, through the last p-trigger / n-trigger node of DT_SCR, to ground instead of the expected current path. So, with the blocking diodes, the accumulative property in holding voltage for stacked DT_SCR configuration does not exist. Besides, there are two parasitic diodes (Dp_2 and Dn_2) in this ESD protection circuit. The Dp_2 is the source-to-N-well (VDD) parasitic diode in PMOS Mp1. The Dn_2 is the source-to-P-sub (VSS) parasitic diode in NMOS Mn2.

In the normal circuit operating conditions with VDD and VSS power supplies, the gates of Mp1 and Mp2 (Mn1 and Mn2) are biased at VDD (VSS). Therefore, the Mp1, Mp2, Mn1, and $\mathrm{Mn} 2$ are all in off state, whenever the input signal is logic high (VDD) or logic low (VSS). The p-trigger (n-trigger) nodes of the stacked DT_SCR devices are kept at VSS (VDD) through the parasitic resistors (R_well and R_sub), so such stacked DT_SCR devices are guaranteed to be kept off in the normal circuit operating conditions. 
An ESD event zapping on a pad may have the positive or negative voltage with reference to grounded VDD or VSS, so there are four modes of ESD stresses at each I/O pad. The four modes of ESD stresses are positive-to-VSS (PS), negative-to-VSS (NS), positive-to-VDD (PD), and negative-to-VDD (ND) modes [12], [13]. To clearly comprehend the ESD current paths under these ESD stresses, the equivalent circuit of the ESD protection circuit designed with the stacked DT_SCR devices for I/O pad is illustrated in Fig. 10. The Dn_1 is the N-well (under the N+ diffusion at the end of the SCR path) to P-sub (VSS) parasitic diode in DT_SCR_4. The Dp_1 is the $\mathrm{P}+$ to $\mathrm{N}$-well (connected to VDD) parasitic diode in the DT_SCR_1.

Under the PS-mode ESD-zapping condition (with grounded VSS but floating VDD), the gates of Mp1 and Mp2 are initially floating with a zero voltage level, thereby the Mp1 and Mp2 will be turned on due to the positive ESD voltage on the pad. So, the Mp1 will conduct some initial ESD current into the p-trigger nodes of the two stacked DT_SCR devices between I/O pad and VSS pin. Synchronously, the Mp2 will draw some initial ESD current out from the n-trigger nodes of the stacked DT_SCR devices. The Vt1 of the two stacked DT_SCR devices will be reduced to a low voltage level, therefore the two stacked DT_SCR devices can be quickly triggered on. So, the ESD current can be discharged from I/O pad to the grounded VSS through the stacked DT_SCR devices. However, the gate voltages of Mp1 and Mp2 may be charged up by the ESD energy through the forward-biased diodes Dp_1 and Dp_2, so the R1C1 time constant is designed to keep the gates of Mp1 and Mp2 at a relatively low voltage level. Then, the voltage pulses can be generated at the p-trigger and n-trigger node to successfully trigger on the stacked DT_SCR devices during ESD stress conditions. With the double-triggered technique, the required pulse width to trigger the DT_SCR into latching state can be shortened, as that shown in Fig.6, so the RC time constant can be designed smaller to save the layout area.

Under the ND-mode ESD-zapping condition (with grounded VDD but floating VSS), the gates of Mn1 and Mn2 are initially floating with a zero voltage level, thereby the Mn1 and Mn2 will be turned on due to the negative ESD voltage on the pad. So, the Mn1 will conduct some initial ESD current into the p-trigger nodes of the two stacked DT_SCR devices between I/O pad and VDD pin. Synchronously, the Mn2 will draw some initial ESD current out from the n-trigger nodes of the stacked DT_SCR devices. Therefore, the two stacked DT_SCR devices will be triggered on, and the ESD current can be discharged from I/O pad to the grounded VDD through the stacked DT_SCR devices. Furthermore, the R2C2 time constant is also designed to avoid the 
gate voltages of Mn1 and Mn2 being charged up quickly through the parasitic diodes Dn_1 and Dn_2.

Under the NS-mode (PD-mode) ESD-zapping condition, the parasitic diodes Dn_1 and Dn_2 (Dp_1 and Dp_2) will be forward biased and turned on to discharge the ESD current from I/O pad to the grounded VSS (VDD). The four modes (PS, NS, PD, and ND) of ESD stresses can be clamped to a very low voltage level by the stacked DT_SCR devices or the forward-biased parasitic diodes, so the thinner gate oxide in deep sub-quarter-micron CMOS technologies can be fully protected. The diode in forward-biased condition can often sustain a much high ESD level. The ESD level of an I/O pad is dominated by the weakest ESD current path, so the experimental measurements in the following will be focused on the PS-mode or ND-mode ESD-zapping conditions.

HSPICE is used to verify the functions of ESD-detection circuits on the ESD protection circuit for the I/O pad. The transient simulation on the ESD-detection circuits in Fig. 9 under PS-mode and ND-mode ESD zapping conditions are shown in Figs. 11(a) and 11(b), respectively, where $\mathrm{R} 1=\mathrm{R} 2=100 \mathrm{k} \Omega, \mathrm{C} 1=\mathrm{C} 2=1 \mathrm{pF}$, and the device dimensions $\mathrm{W} / \mathrm{L}$ of $\mathrm{Mp} 1, \mathrm{Mp} 2, \mathrm{Mn} 1$, and Mn2 are $10 \mu \mathrm{m} / 0.25 \mu \mathrm{m}, 20 \mu \mathrm{m} / 0.25 \mu \mathrm{m}, 10 \mu \mathrm{m} / 0.25 \mu \mathrm{m}$, and $5 \mu \mathrm{m} / 0.25 \mu \mathrm{m}$. Because the overdrive voltage (Vsg) of Mp2 is smaller than that of Mp1 under the same pad voltage, the Mp2 in Fig. 9 is designed with larger channel width than that of Mp1. In Fig. 11(a), when a 0-to-8 V voltage pulse with a rise time of $10 \mathrm{~ns}$ is applied to the $\mathrm{I} / \mathrm{O}$ pad of Fig. 9, the substrate-triggered and well-triggered currents can be synchronously generated by the ESD-detection circuit, which is formed by R1, C1, Mp1, and Mp2, to trigger on the stacked DT_SCR devices. In Fig. 11(b), the substrate-triggered and well-triggered currents can be also synchronously generated by the ESD-detection circuit, which is formed by R2, C2, Mn1, and Mn2, when a 0-to-(-8) V negative voltage pulse with a fall time of 10ns is applied to the $\mathrm{I} / \mathrm{O}$ pad of Fig. 9. Because of the difference of the overdrive voltage between Mn1 and Mn2, the Mn1 in Fig. 9 is designed with larger channel width than that of Mp1. From the simulation results in Fig. 11, the trigger currents at the p-trigger and n-trigger node can be generated almost following the voltage pulse on the I/O pad. The delay resulting from the ESD-detection circuit in Fig. 9 can be almost neglectable. The triggered currents, which are the function of resistance $(\mathrm{R} 1, \mathrm{R} 2)$, capacitance $(\mathrm{C} 1, \mathrm{C} 2)$, and device dimensions of PMOS devices and NMOS devices, can be fine tuned by HSPICE simulator to fit the practical applications in different CMOS processed. 


\section{B. ESD Clamp Circuit between the Power Rails}

The stacked DT_SCR devices can be also applied to design the power-rail ESD clamp circuit. The VDD-to-VSS ESD clamp circuit designed with the two stacked DT_SCR devices is realized in Fig. 12 for the circuit applications of $2.5 \mathrm{~V}$. The function of the ESD-detection circuit, which is formed with resistor (R), capacitor (C), and inverters (inv_1 and inv_2), is to distinguish VDD power-on event (with a rise time of $\sim \mathrm{ms}$ ) or ESD-stress events (with a rise time of $\sim \mathrm{ns}$ ) [14]. During normal VDD power-on transition (from low to high), the input of the inv_1 can follow up in time with the power-on VDD waveform, so the output of the inv_1 (or the input of the inv_2) will be biased at zero. Therefore, the output of the inv_2 will be kept at VDD. The p-trigger / n-trigger nodes of stacked DT_SCR devices are biased at VSS / VDD in this situation, so the two stacked DT_SCR devices are kept off and do not interfere with the normal circuit operating functions.

When a positive ESD voltage is applied to VDD with VSS relatively grounded, the RC delay will keep the input of the inv_1 at a relatively low voltage level for a long time. Therefore, the output of the inv_1 (or the input of the inv_2) will become high, and then the output of the inv_2 will be kept at a low voltage level. Thus, the p-trigger current and n-trigger current voltage pulses can be synchronously generated to trigger on the two stacked DT_SCR devices. ESD current is discharged from VDD to VSS through the stacked DT_SCR devices. When a negative ESD voltage is applied to VDD with VSS relatively grounded, the negative ESD current can be discharged through the forward-biased P-sub (VSS)-to-N-well (which is connected to VDD) parasitic diode in the ESD protection circuit.

\section{ESD Robustness}

The human-body-model (HBM) and machine-model (MM) ESD stresses are applied to the ESD protection circuits to verify their ESD robustness. The HBM ESD test results on the stacked DT_SCR devices in the device level (without ESD-detection circuit) and the circuit level (with ESD-detection circuit) are compared in Fig. 13. In these ESD verifications, the failure criterion is defined as the leakage current of the device or circuit after ESD stresses is greater than $1 \mu \mathrm{A}$ under the voltage bias of $2.5 \mathrm{~V}$. For device level, the HBM ESD levels of the 2DT_SCR, 3DT_SCR, and 4DT_SCR (without ESD-detection circuit) are 7, 4, and $1.5 \mathrm{kV}$, respectively. In the layout, each DT_SCR device in the stacked configuration is close to save the layout area, so 
the power (thermal) dissipation among the stacked DT_SCR devices will interact to reduce the ESD robustness of stacked DT_SCR devices. From other aspect, because the total holding voltage of stacked DT_SCR configuration is increased with the increase of the number of the stacked DT_SCR devices, the HBM ESD robustness of the stacked DT_SCR devices is decreased due to power $=\mathrm{I}_{\mathrm{ESD}} \times \mathrm{V}_{\text {hold }}$. But, the ESD levels of the stacked DT_SCR devices can be greatly improved for the 3DT_SCR or 4DT_SCR, if the desired ESD-detection circuit is used to trigger the stacked DT_SCR devices on. From Fig. 13, the ESD levels of the stacked DT_SCR devices with ESD-detection circuit are all boosted up to $>8 \mathrm{kV}$. The measurement results on the MM ESD levels of the stacked DT_SCR devices with or without ESD-detection circuit are shown in Fig. 14. The MM ESD level is also decreased when the number of stacked DT_SCR devices is increased. However, the MM ESD levels of the stacked DT_SCR devices can be also improved if the desired ESD-detection circuit is used to trigger the stacked DT_SCR devices on. The MM ESD levels of the 2DT_SCR, 3DT_SCR, and 4DT_SCR (with ESD-detection circuit) are 700,525 , and $375 \mathrm{~V}$, respectively.

A gate-grounded NMOS (GGNMOS) device with W/L of $200 \mu \mathrm{m} / 0.5 \mu \mathrm{m}$ had been also fabricated in the same CMOS process with extra silicide-blocking mask for comparison reference. Such GGNMOS occupied a large active layout area of $25.8 \mu \mathrm{m} \times 50 \mu \mathrm{m}$ can sustain the HBM ESD level of $3.5 \mathrm{kV}$. For the ESD protection circuit designed with 2DT_SCR and ESD-detection circuit, the HBM (MM) ESD level per layout area is $>10 \mathrm{~V} / \mu \mathrm{m}^{2}\left(0.88 \mathrm{~V} / \mu \mathrm{m}^{2}\right)$, but it is only $2.71 \mathrm{~V} / \mu \mathrm{m}^{2}\left(0.29 \mathrm{~V} / \mu \mathrm{m}^{2}\right)$ for the GGNMOS. This has verified the excellent area efficiency of the ESD protection circuits realized with the DT_SCR devices.

By using the transmission line pulsing (TLP) measurement [15], [16], the secondary breakdown current (It2) of the DT_SCR device can be found. The It2 is another index for the HBM ESD robustness, which is indicated in this work by the sudden increase of the leakage current at the voltage bias of $2.5 \mathrm{~V}$. The relation between second breakdown current (It2) and HBM ESD level $\left(\mathrm{V}_{\mathrm{ESD}}\right)$ can be approximated as:

$$
\mathrm{V}_{\mathrm{ESD}} \cong(1500+\mathrm{Ron}) \times \mathrm{It} 2 \text {, }
$$

where Ron is the dynamic turn-on resistance of the device under test. The TLP-measured FV curves of the two stacked DT_SCR devices with or without ESD-detection circuit depicted in Fig. 12 are shown in Fig. 15. The stacked DT_SCR devices with ESD-detection circuit can be triggered on at a lower voltage level of $\sim 2 \mathrm{~V}$, however, the stacked DT_SCR devices without 
ESD-detection circuit can not be triggered on until a higher voltage level of $\sim 30 \mathrm{~V}$. Moreover, the It2 of the stacked DT_SCR device with ESD-detection circuit can be improved, which is in accordance with the results in Fig. 13. This has confirmed that the ESD-detection circuit proposed in this paper can indeed reduce the switching voltage of DT_SCR and enhance its ESD robustness.

\section{Turn-on Verification}

In order to verify the function of the ESD protection circuit in Fig. 12, a voltage pulse with a pulse width of $400 \mathrm{~ns}$ and rise time of $10 \mathrm{~ns}$ is applied to VDD of Fig. 12 with grounded VSS. In Fig. 16, a 0-to-5 V voltage pulse applied on the VDD pin is clamped to $\sim 3 \mathrm{~V}$ by the turned-on 2DT_SCR. This implies that the ESD-detection circuit realized with the R, C, inv_1, and inv_2 can indeed generate the required double-triggered currents. The stacked DT_SCR devices can be successfully triggered into latching state without involving the junction avalanche breakdown mechanism. The clamped voltage of $\sim 3 \mathrm{~V}$ has also verified that the proposed ESD protection circuits with two stacked DT_SCR devices are free to latchup issue under normal operating conditions.

\section{CONClusion}

The novel DT_SCR device used for on-chip ESD protection circuits has been successfully investigated in a $0.25-\mu \mathrm{m}$ salicided CMOS process. With both the substrate and $\mathrm{N}$-well triggered currents, the switching voltage and turn-on time of DT_SCR device can be successfully reduced to only $\sim 1.5 \mathrm{~V}$ and $\sim 10 \mathrm{~ns}$, respectively. For IC applications with VDD of $2.5 \mathrm{~V}$, the ESD protection circuits designed with two DT_SCR devices in stacked configuration and ESD-detection circuits have a clamp voltage of $\sim 3 \mathrm{~V}$, which are free to latchup issue. Such ESD

protection circuits can sustain the HBM (MM) ESD level per area of $>10 \mathrm{~V} / \mu \mathrm{m}^{2}\left(0.88 \mathrm{~V} / \mu \mathrm{m}^{2}\right)$ in a $0.25-\mu \mathrm{m}$ fully salicided CMOS process without using extra process modification. 


\section{REFERENCES}

[1] R. Rountree, "ESD protection for submicron CMOS circuits: issues and solutions," in IEDM Tech. Dig., 1988, pp. 580-583.

[2] M.-D. Ker, "Electrostatic discharge protection circuits in CMOS IC' s using the lateral SCR devices: an overview," in Proc. IEEE Int. Conf. on Electronics Circuits and Systems, 1998, pp. 325-328.

[3] M. Corsi, R. Nimmo, and F. Fattori, "ESD protection of BiCMOS integrated circuits which need to operate in the harsh environments of automotive or industrial," in Proc. EOS/ESD Symp., 1993, pp. 209-213.

[4] G. Notermans, F. Kuper, and J. M. Luchis, "Using an SCR as ESD protection without latch-up danger," Microelectronics Reliability, vol. 37, pp. 1457-1460, 1997.

[5] M.-D. Ker, "Lateral SCR devices with low-voltage high-current triggering characteristics for output ESD protection in submicron CMOS technology," IEEE Trans. Electron Devices, vol. 45, pp. 849-860, 1998.

[6] A. Chatterjee and T. Polgreen, "A low-voltage triggering SCR for on-chip ESD protection at output and input pads," IEEE Electron Device Letters, vol. 12, pp. 21-22, 1991.

[7] M.-D. Ker and H.-H. Chang, "How to safely apply the LVTSCR for CMOS whole-chip ESD protection without being accidentally triggered on," Journal of Electrostatics, vol. 47, pp. 215-248, 1999.

[8] C. Russ, M. Mergens, J. Armer, P. Jozwiak, G. Kolluri, L. Avery, and K. Verhaege, "GGSCR: GGNMOS triggered silicon controlled rectifiers for ESD protection in deep submicron CMOS processes," in Proc. EOS/ESD Symp., 2001, pp. 22-31.

[9] M.-D. Ker and C.-Y. Wu, "Modeling the positive-feedback regenerative process of CMOS latchup by a positive transient pole method-part I: theoretical derivation," IEEE Trans. Electron Devices, vol.42, pp. 1141-1148, 1995.

[10] R. R. Troutman, Latchup in CMOS technology. Boston, MA: Kluwer, 1986.

[11] S. Krishnan and A. Amerasekera, "Antenna protection strategy for ultra-thin gate MOSFETs," in Proc. IEEE Int. Reliability Physics Symp., 1998, pp. 302-306.

[12] M.-D. Ker, C.-Y. Wu, and H.-H. Chang, "Complementary-LVTSCR ESD protection circuit for submicron CMOS VLSI/ULSI," IEEE Trans. Electron Devices, vol. 43, pp. 588-598, 1996. 
[13] "ESD Association standard test method for electrostatic discharge sensitivity testing: Human body model-Component level," ESD Association, New York, ESD STM 5.1, 1998.

[14] M.-D. Ker, "Whole-chip ESD protection design with efficient VDD-to-VSS ESD clamp circuits for submicron CMOS VLSI," IEEE Trans. Electron Devices, vol. 46, pp. 173-183, 1999.

[15] T. J. Maloney and N. Khurana, "Transmission line pulsing techniques for circuit modeling of ESD phenomena," in Proc. EOS/ESD Symp., 1985, pp. 49-54.

[16] J. Barth, J. Richner, K. Verhaege, and L. G. Henry, "TLP calibration, correlation, standards, and new techniques," in Proc. EOS/ESD Symp., 2000, pp. 85-96. 


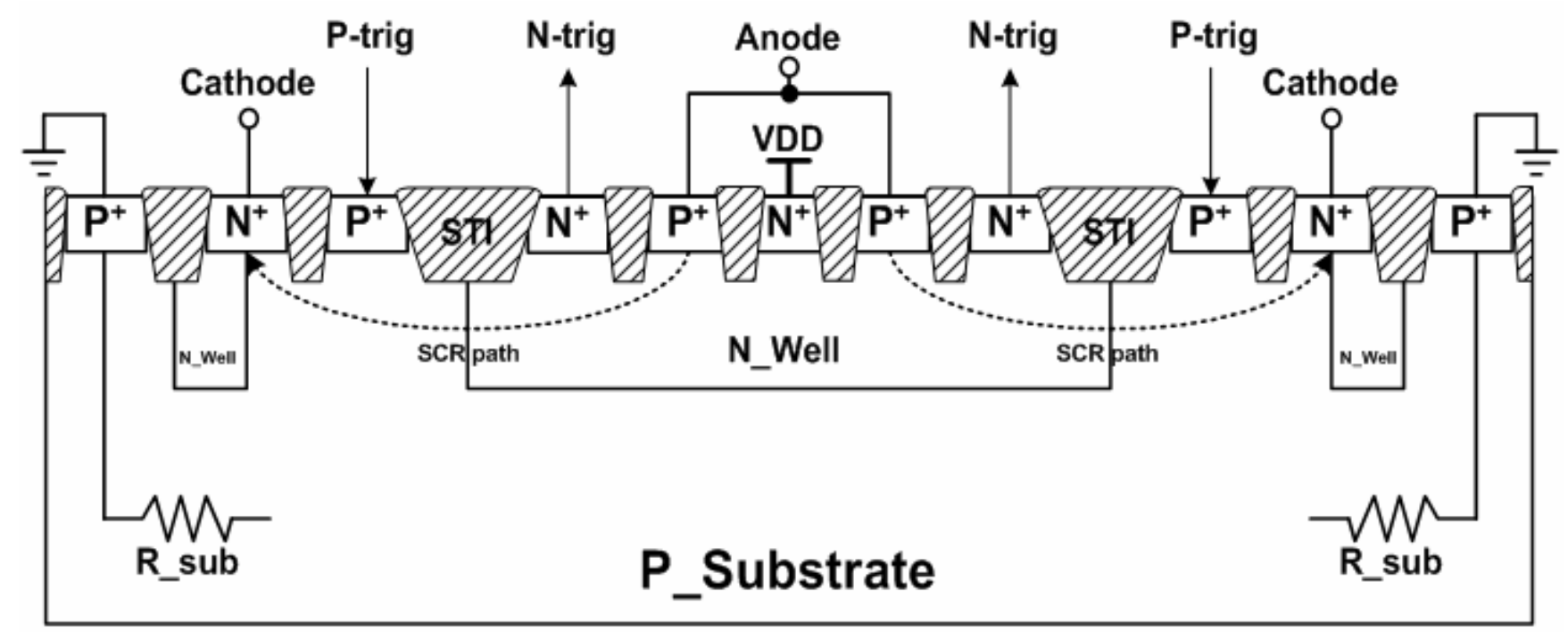

Fig. 1. Device structure of the double-trigger SCR (DT_SCR) device. 


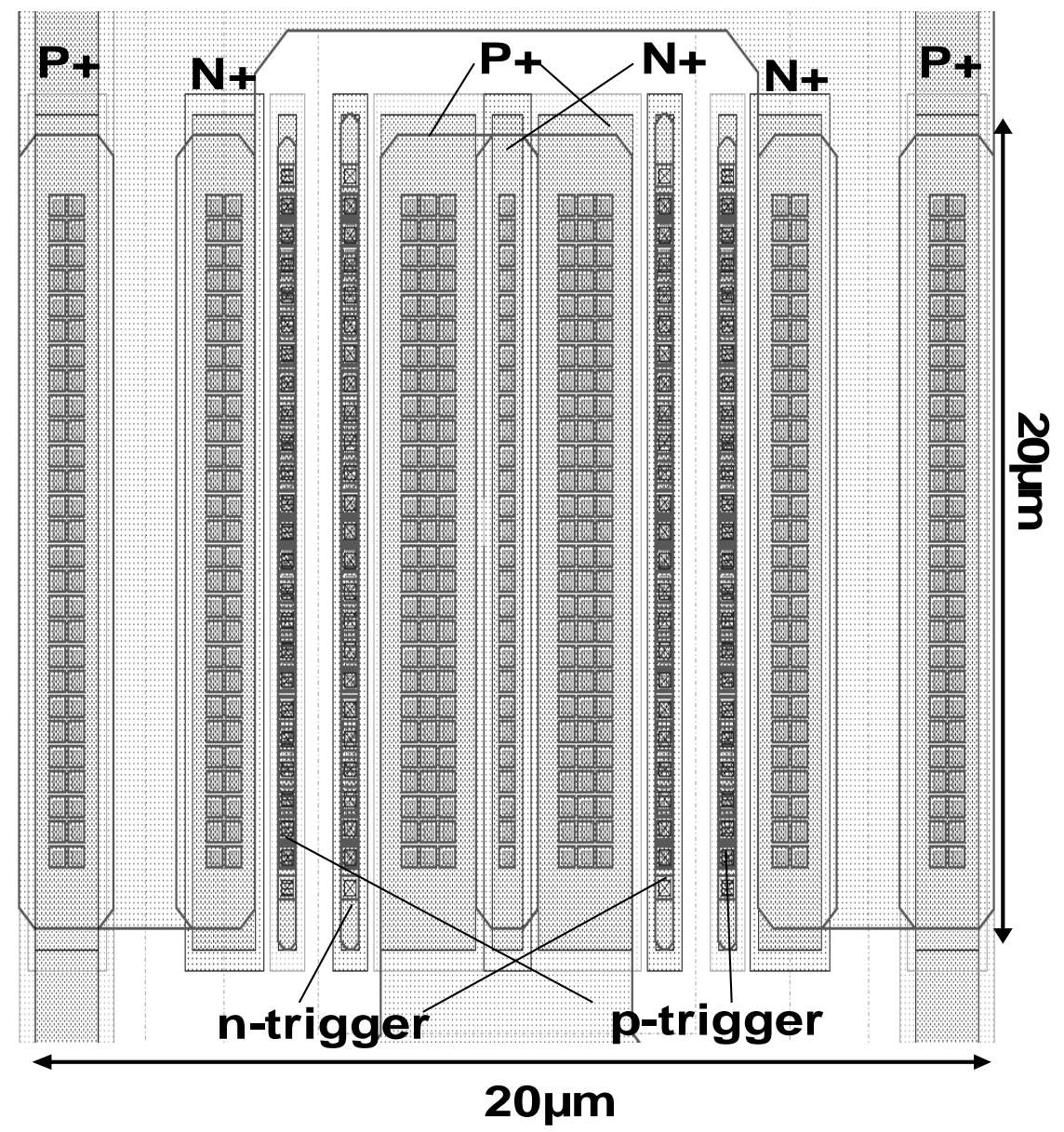

Fig. 2. The layout top view of the DT_SCR device. 


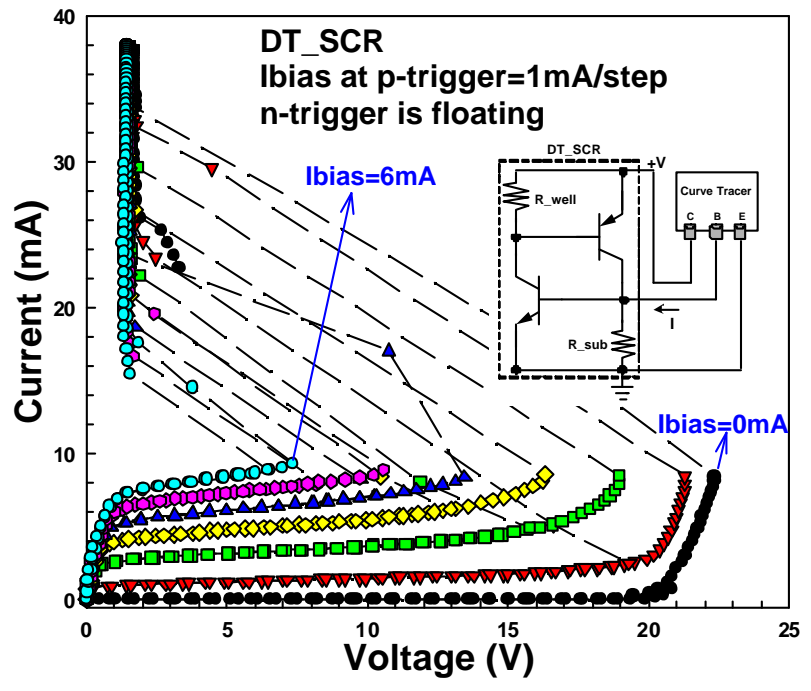

(a)

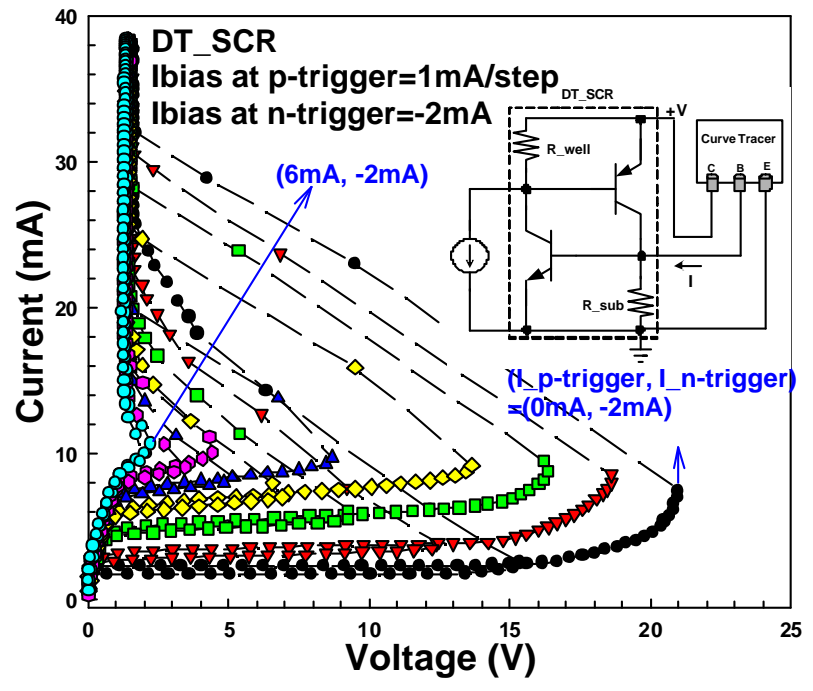

(b)

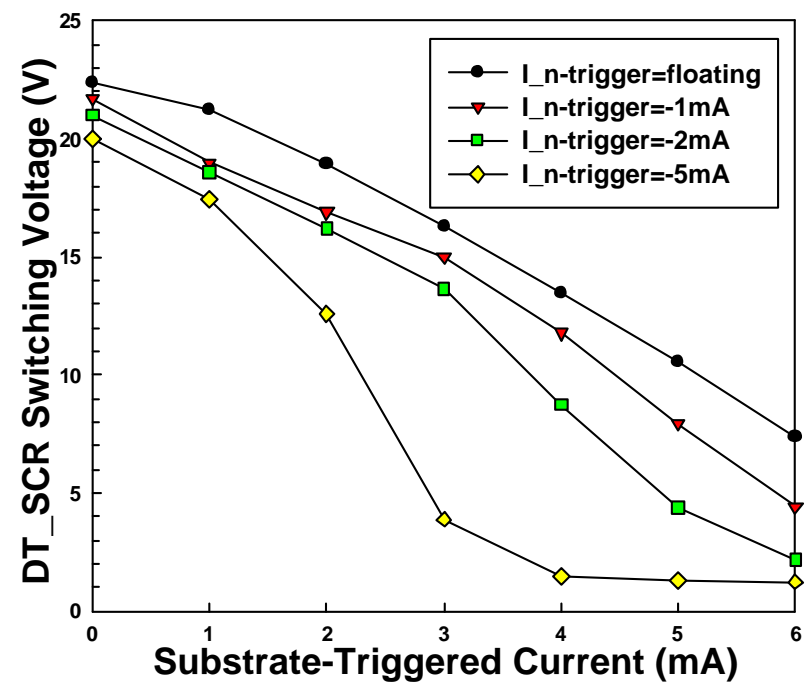

(c)

Fig. 3. (a) The measurement setup and measured DC I-V curves of DT_SCR under different substrate-triggered currents but no $\mathrm{N}$-well triggered current. (b) The measured DC I-V curves of DT_SCR under different substrate-triggered currents and the additional N-well triggered current of -2 $\mathrm{mA}$. (c) The dependence of switching voltage of DT_SCR on substrate-triggered current under different $\mathrm{N}$-well triggered currents. 


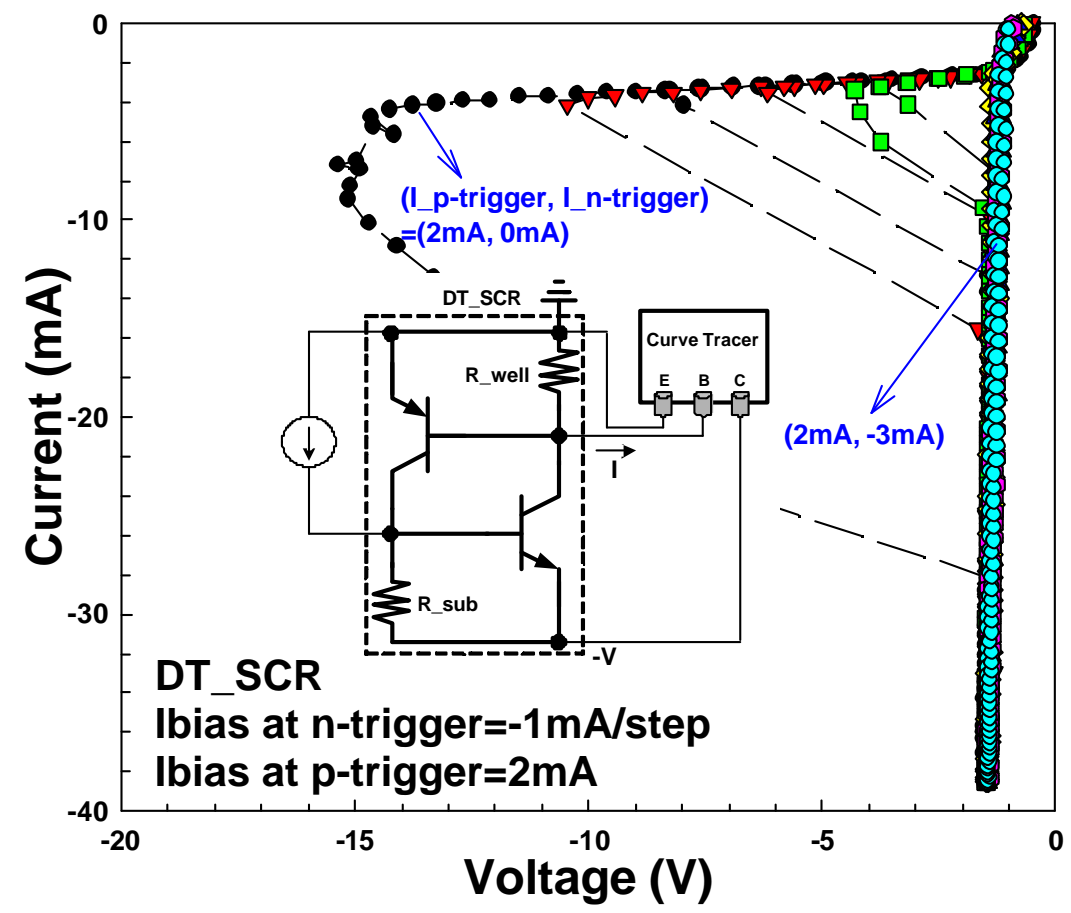

(a)

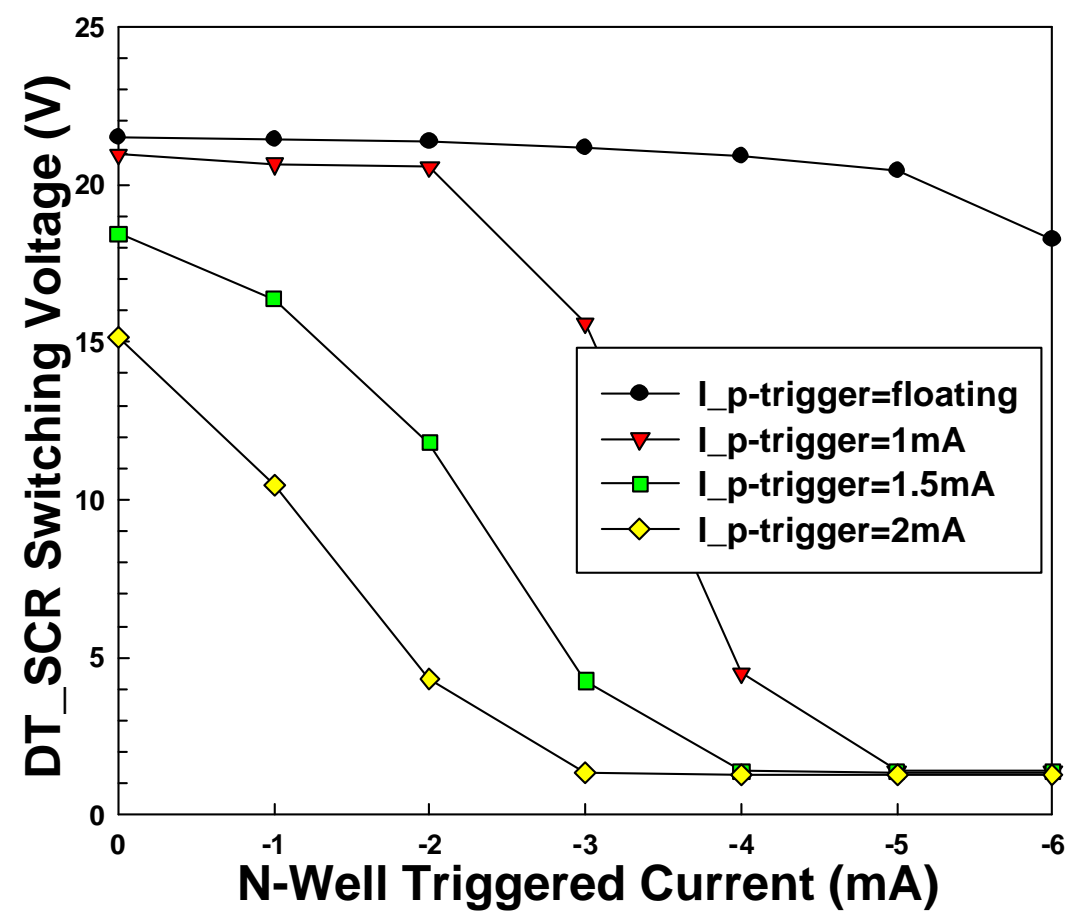

(b)

Fig. 4. (a) The measurement setup and measured DC I-V curves of DT_SCR under different n-trigger currents and the substrate-triggered current of $2 \mathrm{~mA}$. (b) The dependence of switching voltage of DT_SCR on the N-well triggered current under different substrate-triggered currents. 


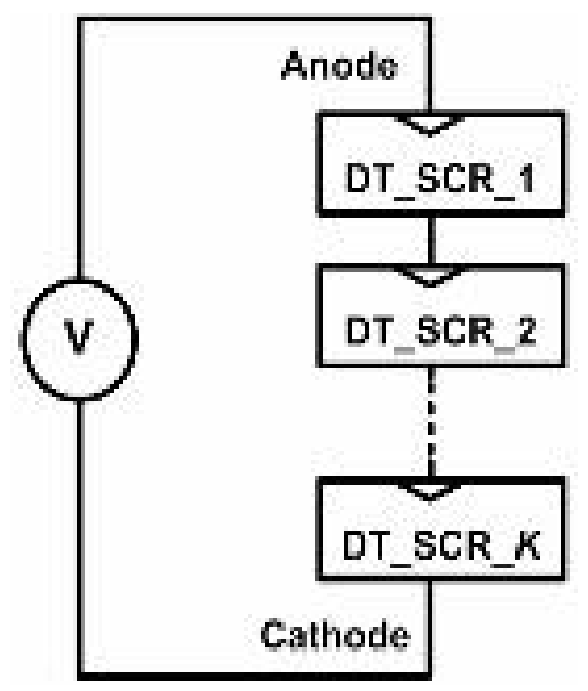

(a)

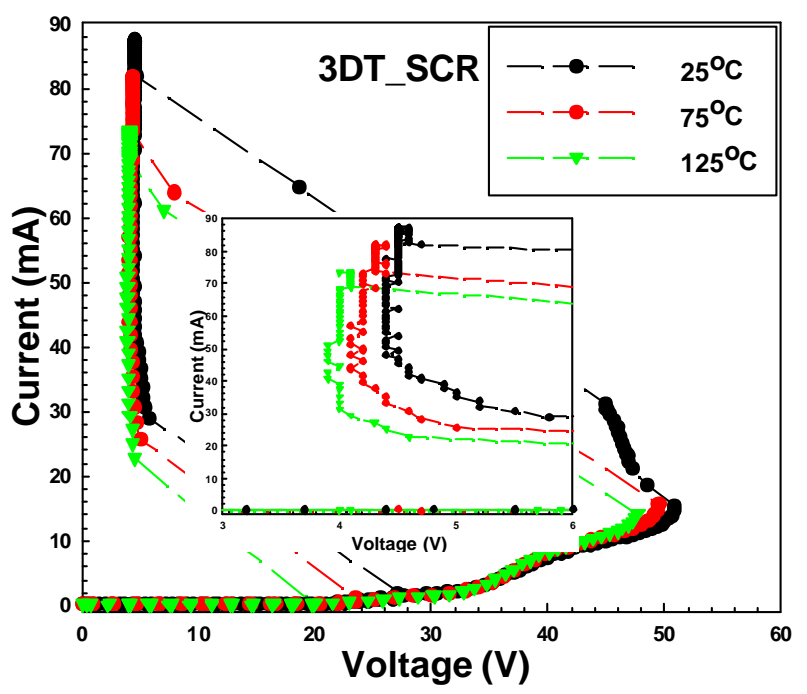

(c)

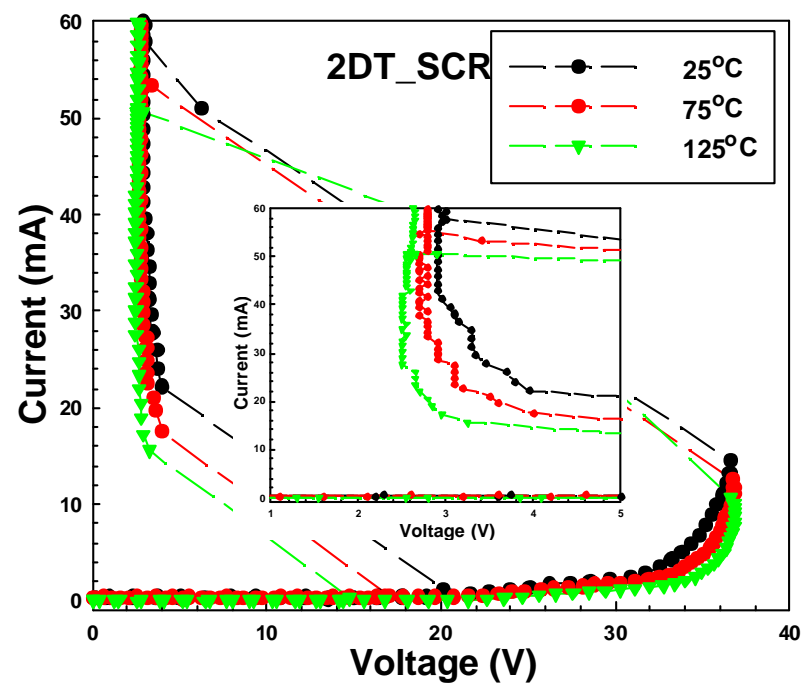

(b)

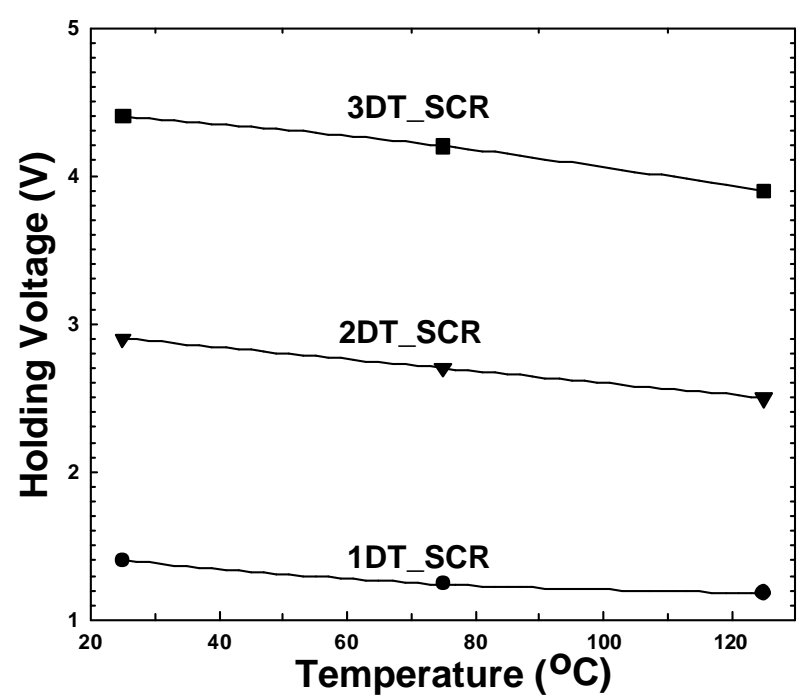

(d)

Fig. 5. The temperature dependence on the total holding voltage of the stacked DT_SCR devices with different stacked numbers. (a) Experimental measurement setup, (b) the measured I-V curves of two DT_SCR devices in stacked configuration (2DT_SCR), (c) the measured I-V curves of three DT_SCR devices in stacked configuration (3DT_SCR), and (d) the relation between the holding voltage and the temperature under different numbers of stacked DT_SCR devices. 


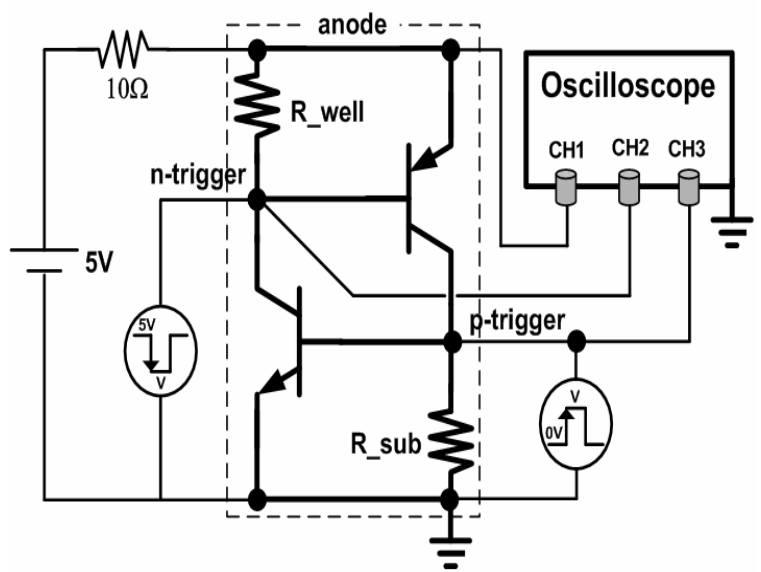

(a)

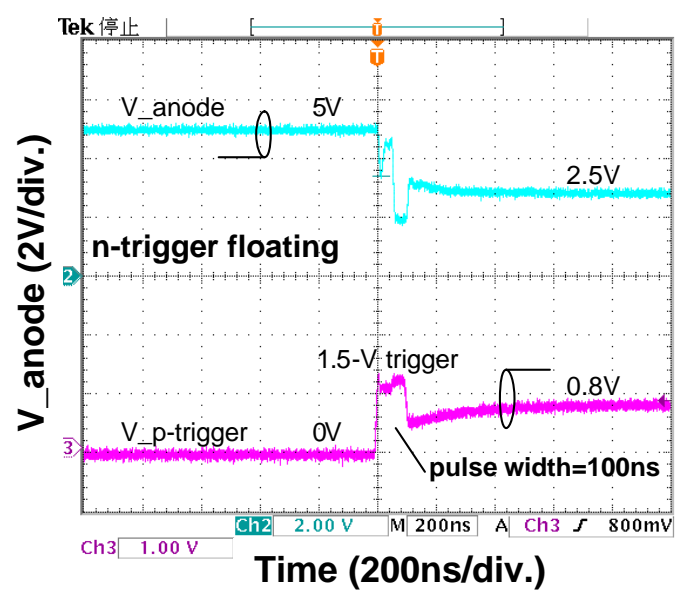

(c)

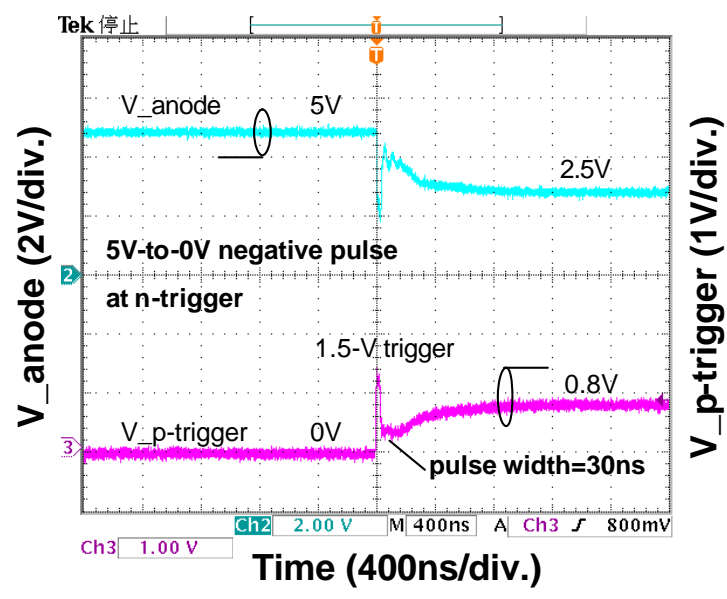

(e)

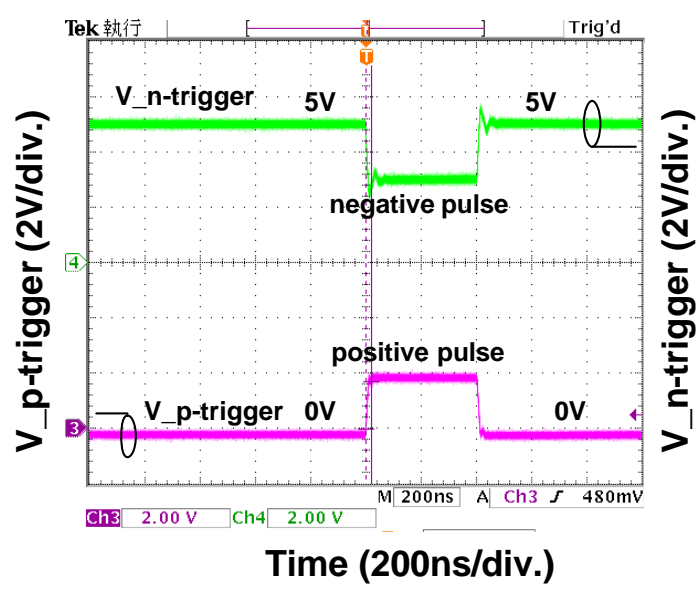

(b)

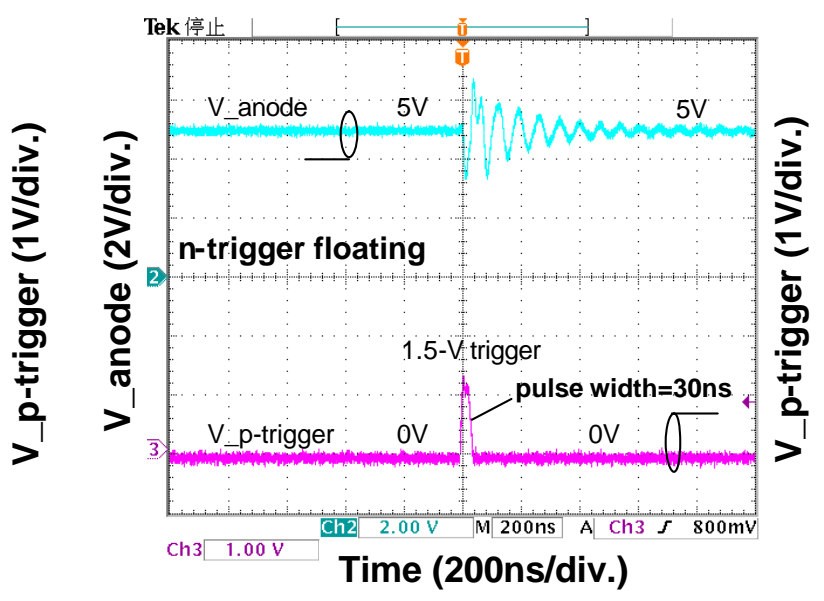

(d)

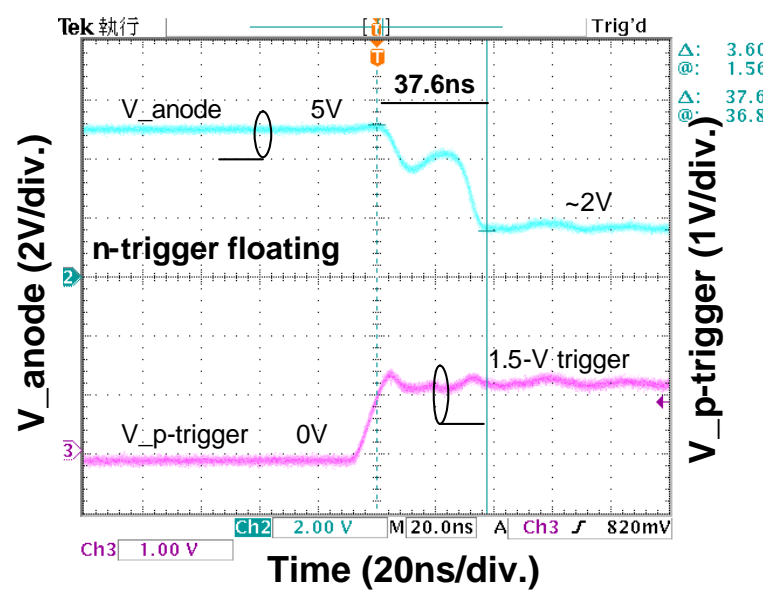

(f) 


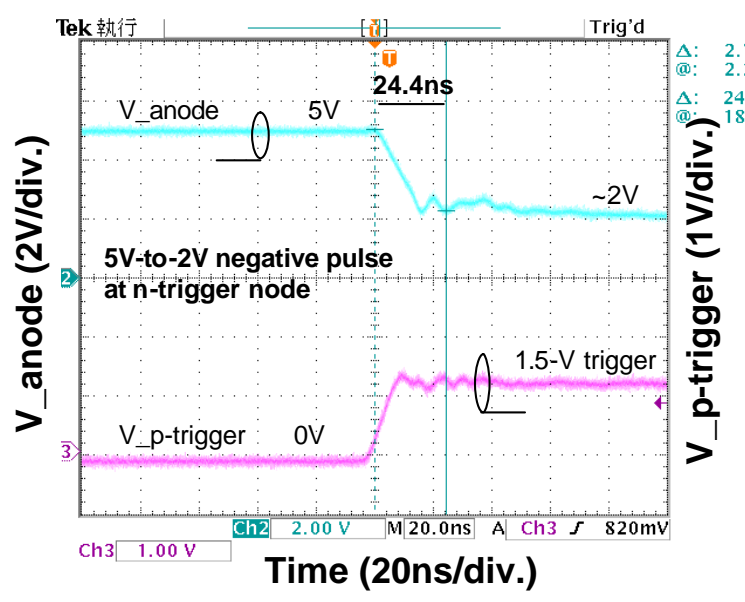

(g)

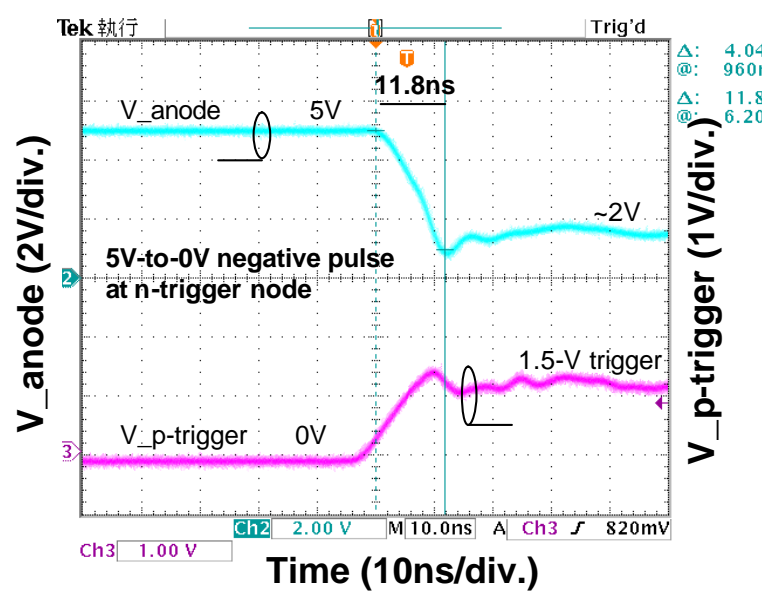

(h)

Fig. 6. The turn-on verification of DT_SCR under different voltage pulses. (a) The measurement setup. (b) Synchronous positive and negative voltage pulses. The measured voltage waveforms on the anode and p-trigger nodes of the DT_SCR device under 1.5-V positive voltage pulse with pulse width of (c) $100 \mathrm{~ns}$, (d) $30 \mathrm{ns,} \mathrm{while} \mathrm{n-trigger} \mathrm{is} \mathrm{floating,} \mathrm{and} \mathrm{(e)} 30 \mathrm{~ns}$ while 5-to-0 V negative voltage pulse is applied to n-trigger. The close-up views of the V_anode at the falling edge while the DT_SCR is synchronously triggering by the $1.5-\mathrm{V}$ positive voltage pulse and under the negative voltage pulse of (f) floating, (g) 5-to-2 V, and (h) 5-to-0 V. 


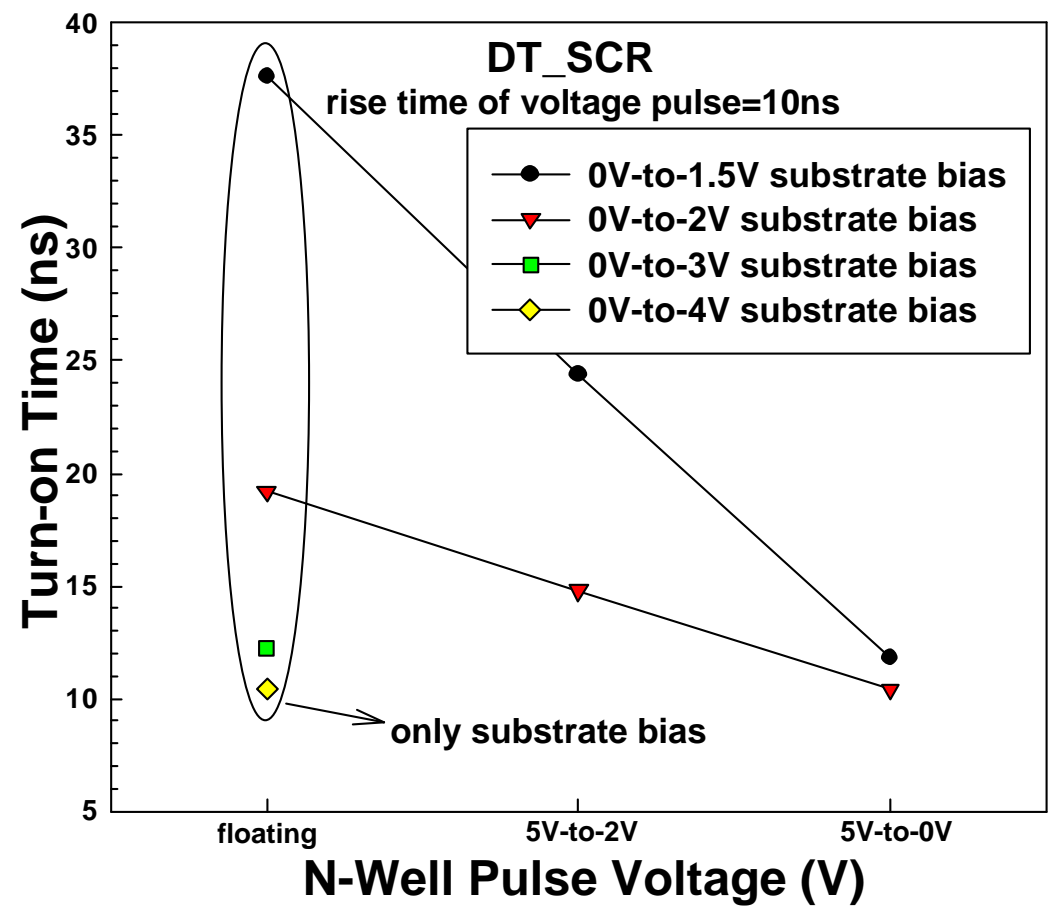

Fig. 7. The dependence of turn-on time of DT_SCR on the N-well biases under different substrate bias conditions with a fixed rise time of $10 \mathrm{~ns}$.

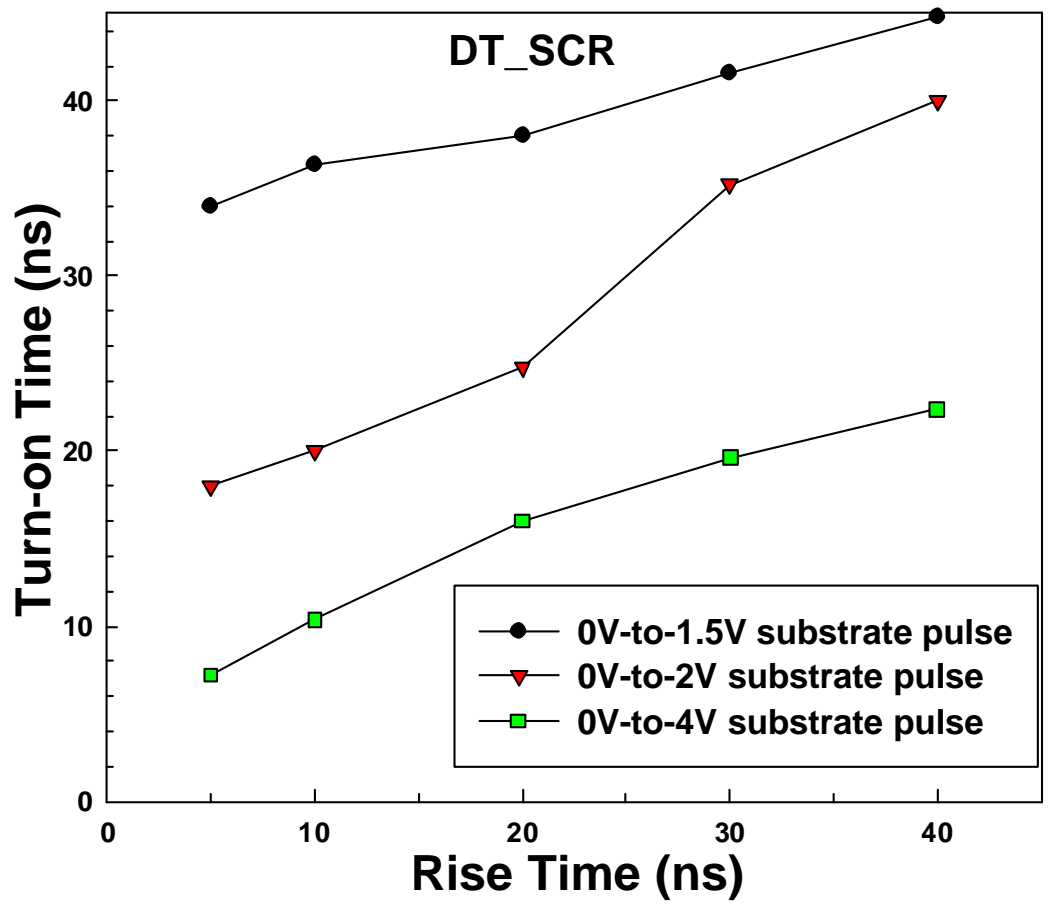

Fig. 8. The dependence of turn-on time of DT_SCR on the rise time of voltage pulse under different substrate bias conditions. 


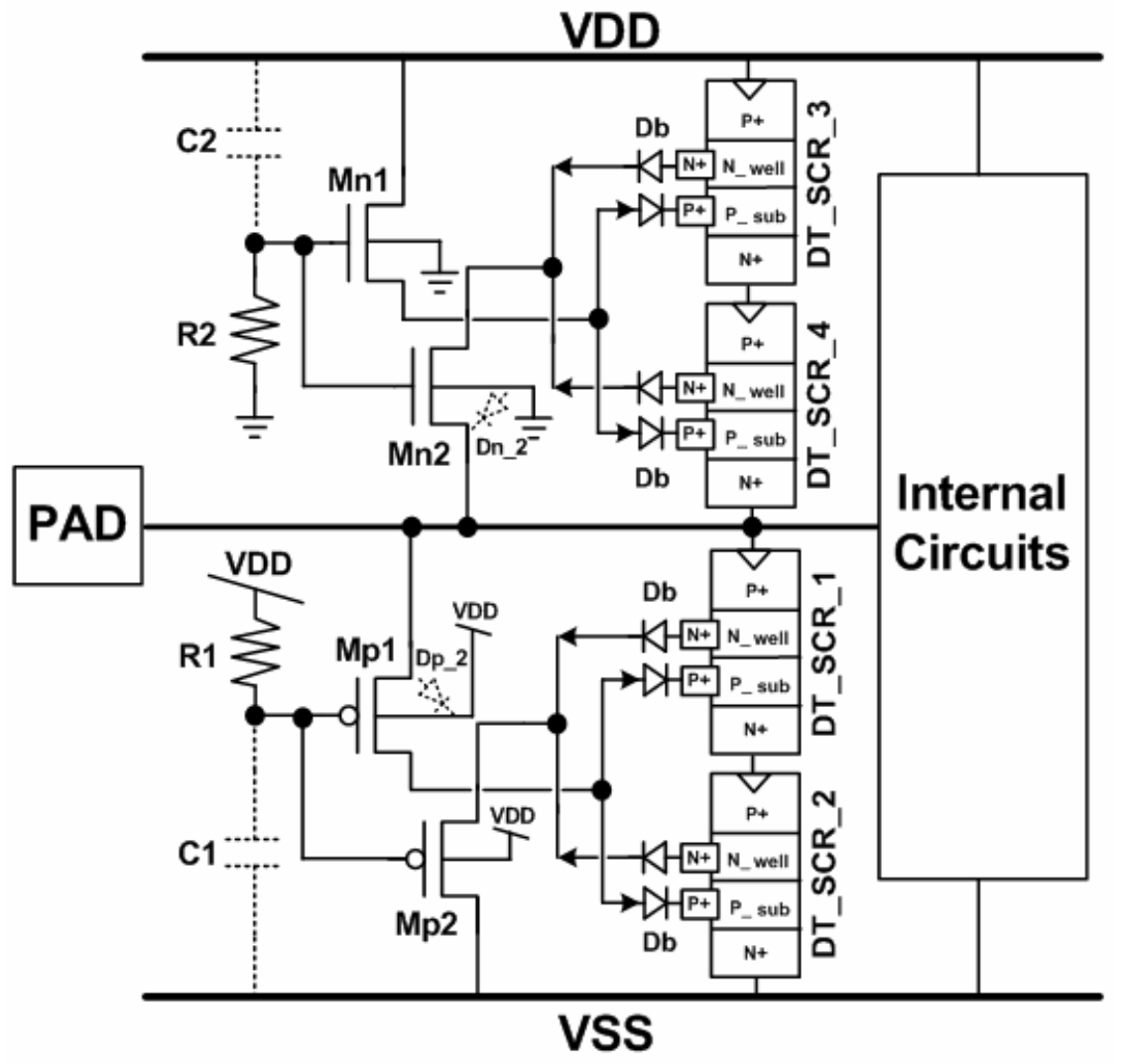

Fig. 9. The ESD protection circuit for the input or output pad with the proposed DT_SCR devices in stacked configuration. 


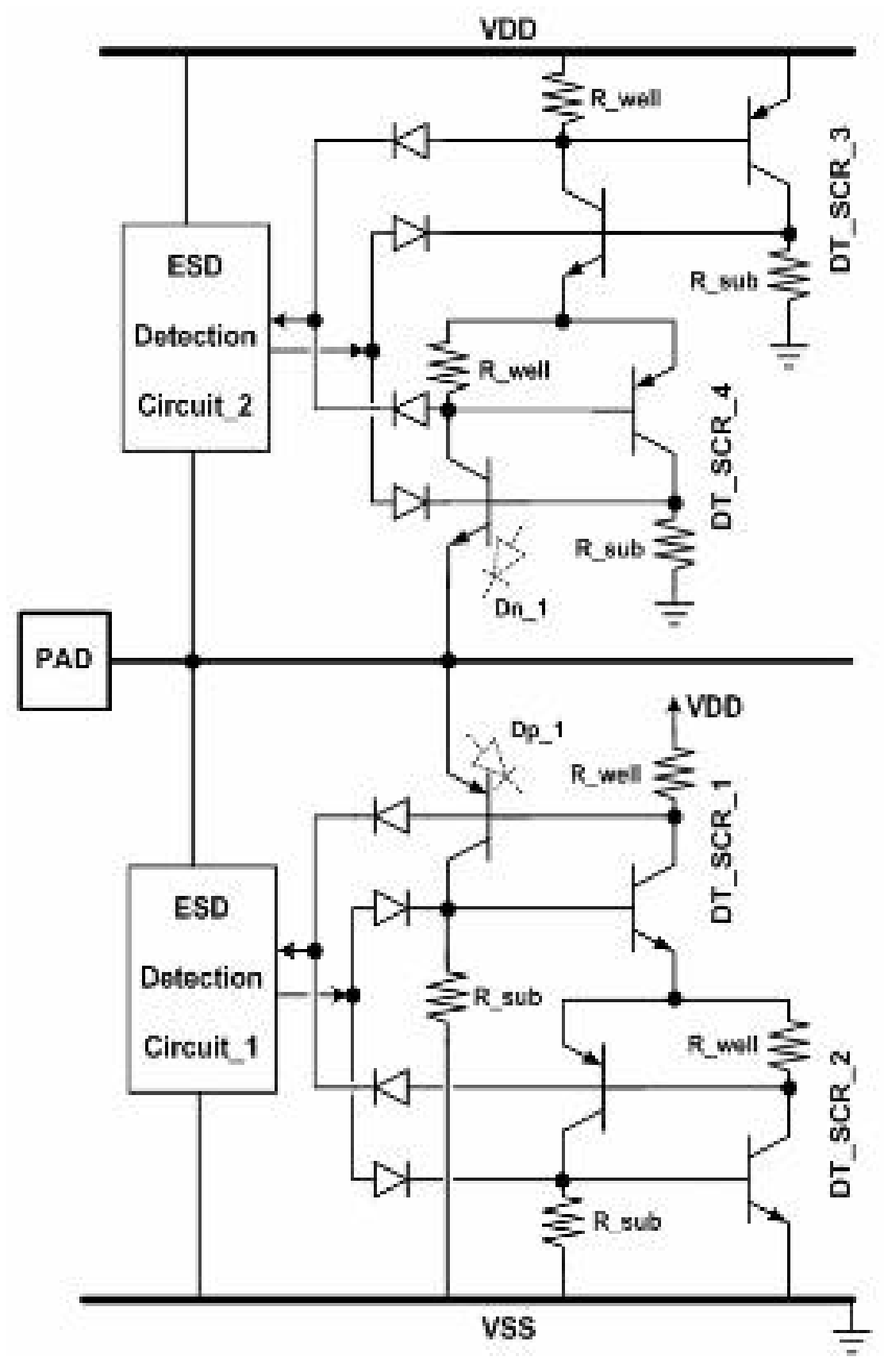

Fig. 10. The equivalent circuit of the stacked DT_SCR devices for the input or output pad. 


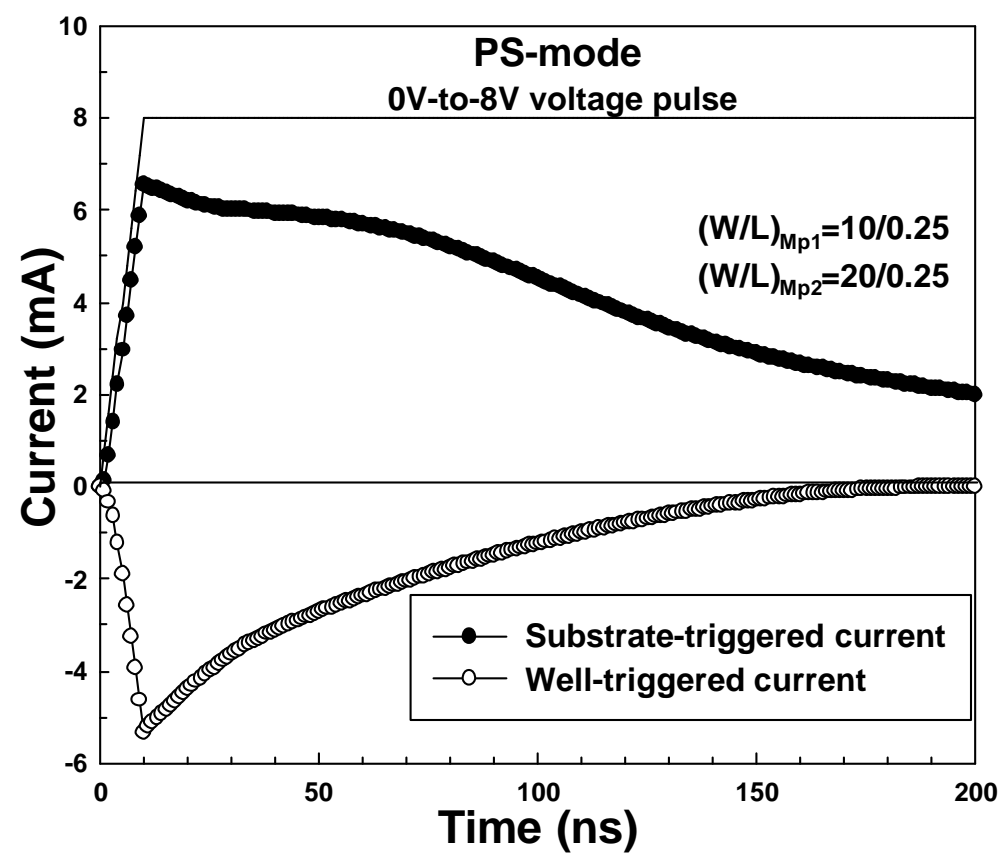

(a)

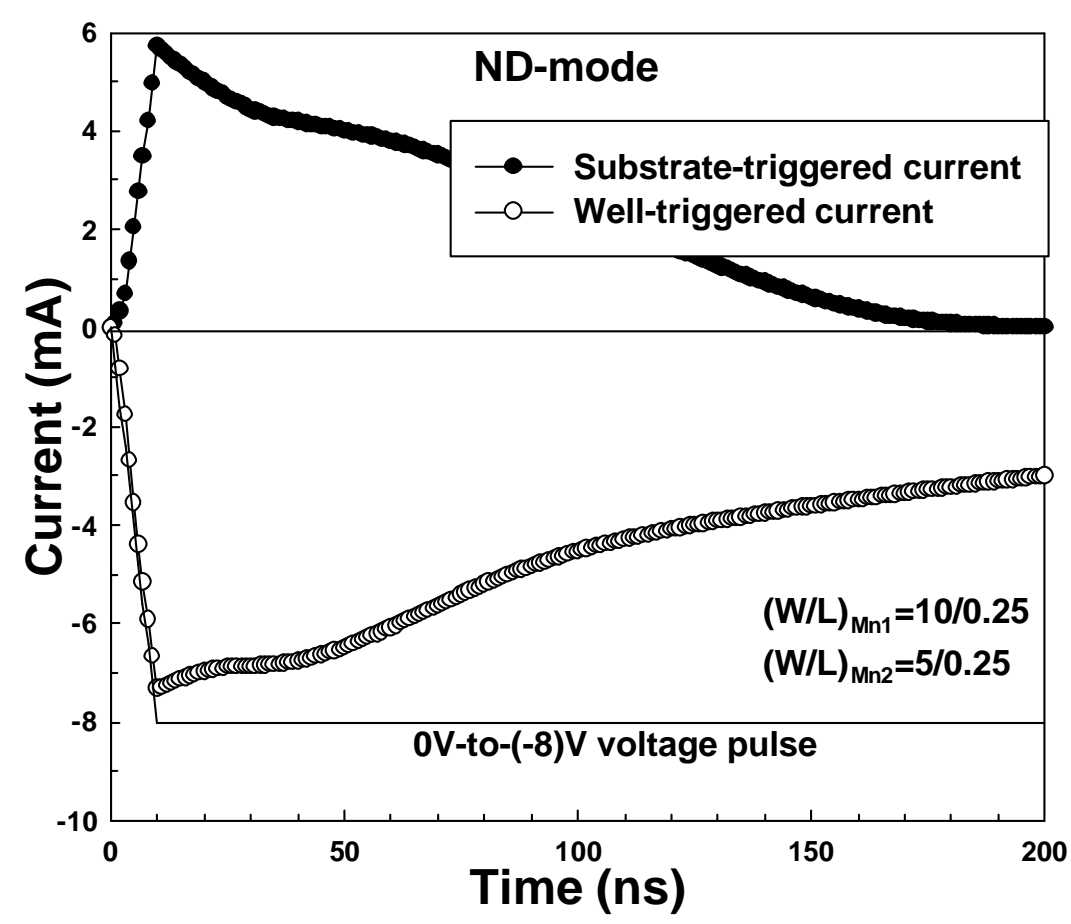

(b)

Fig. 11. HSPICE simulation. The transient simulation on the ESD-detection circuit in Fig. 9 under (a) PS-mode, and (b) ND-mode, ESD-zapping conditions. 


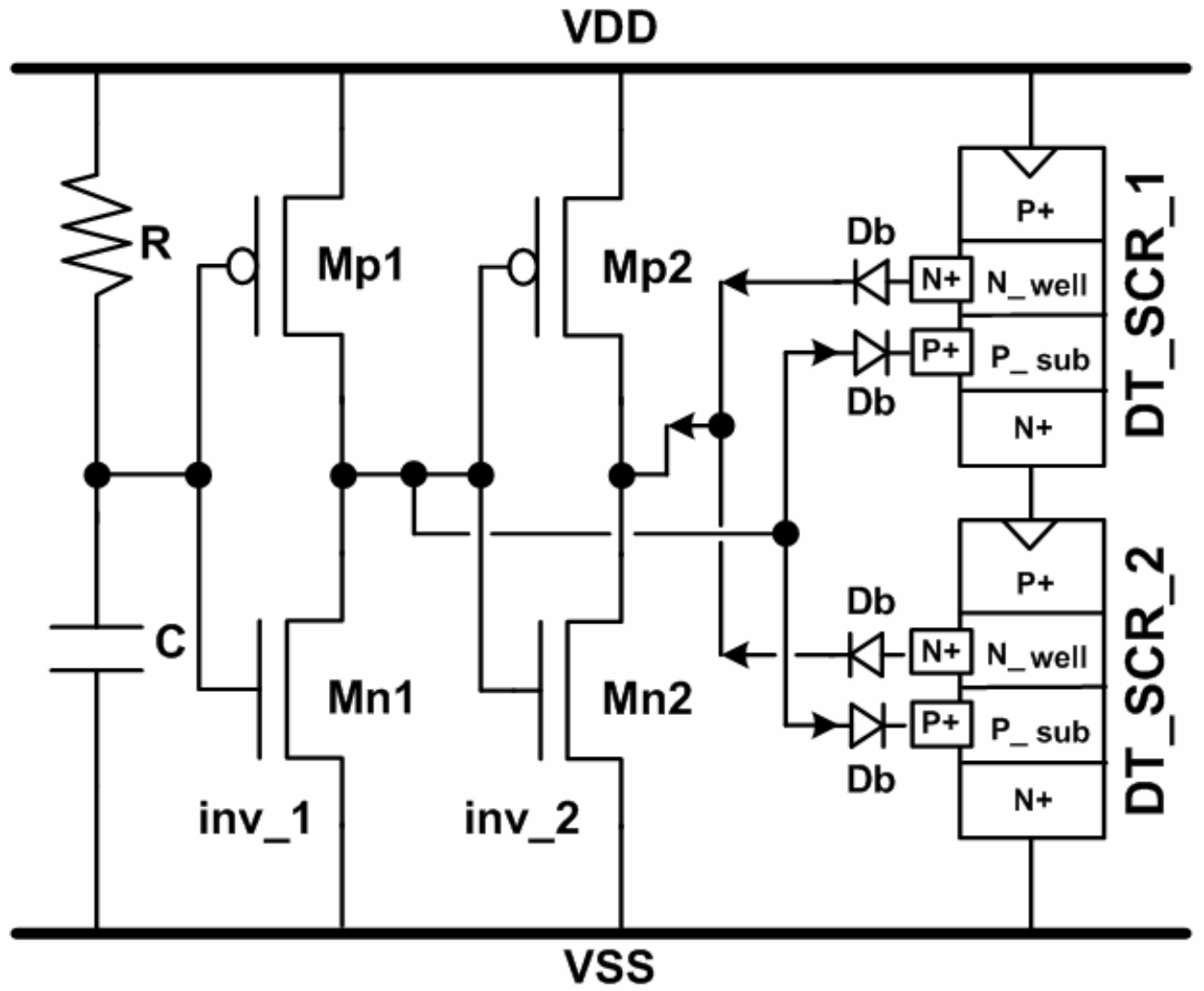

Fig. 12. The power-rail ESD clamp circuit designed with two stacked DT_SCR devices and ESD-detection circuit. 


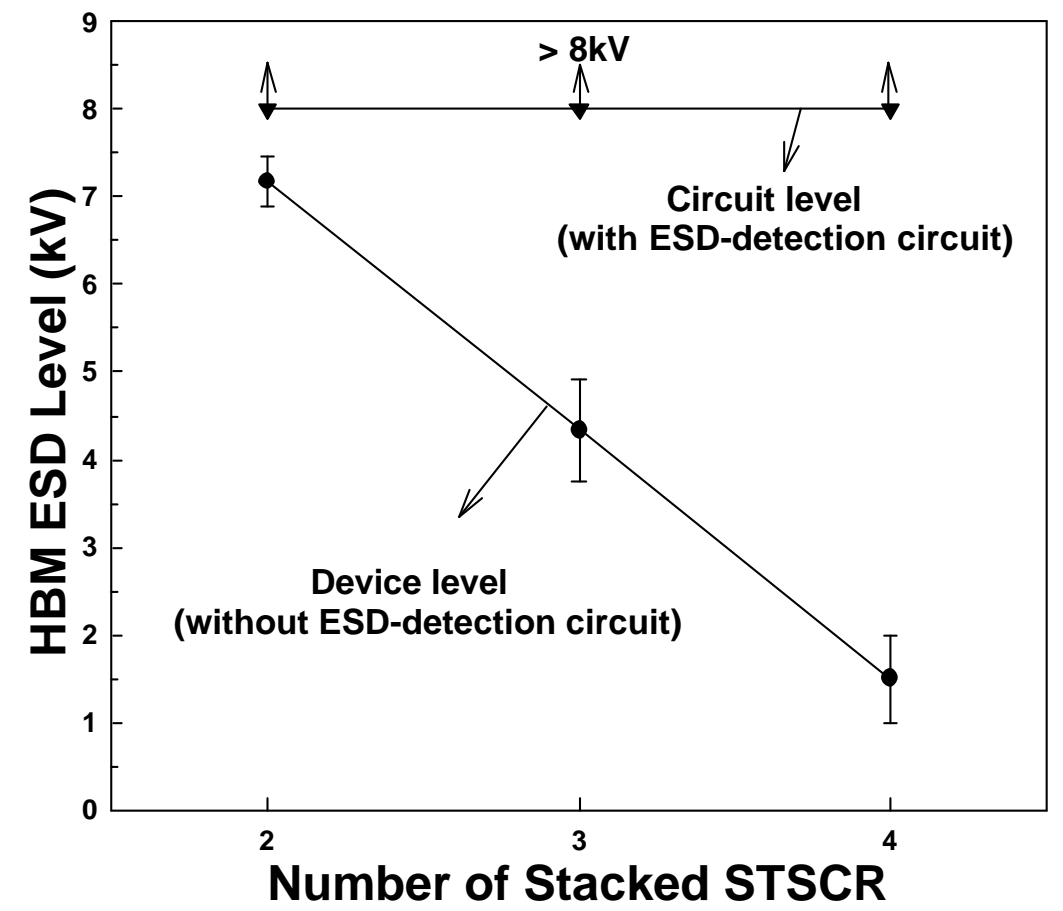

Fig. 13. Dependence of the HBM ESD levels of stacked DT_SCR configuration on the number of the stacked DT_SCR devices (Failure criterion: $\mathrm{I}_{\text {Leakage }}>1 \mu \mathrm{A} @ 2.5 \mathrm{~V}$ bias).

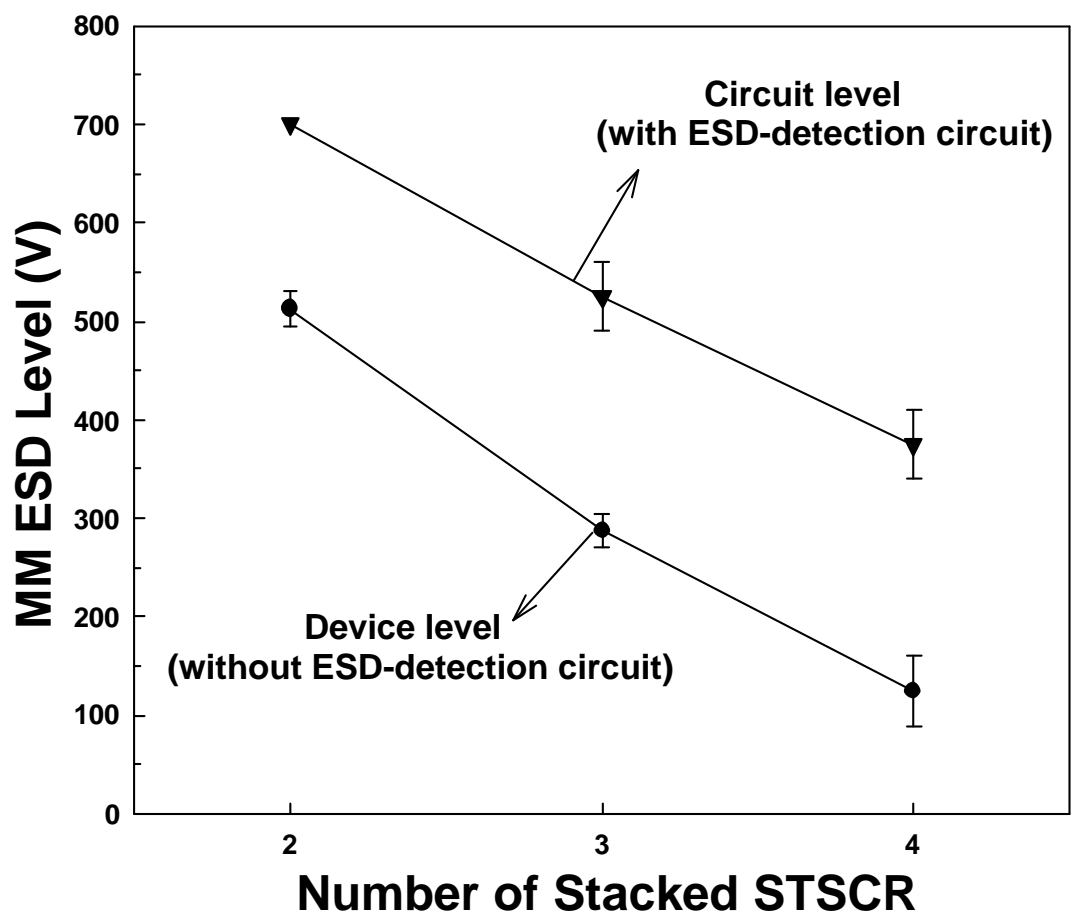

Fig. 14. Dependence of the MM ESD levels of stacked DT_SCR configuration on the number of the stacked DT_SCR devices (Failure criterion: $\mathrm{I}_{\text {Leakage }}>1 \mu \mathrm{A} @ 2.5 \mathrm{~V}$ bias). 


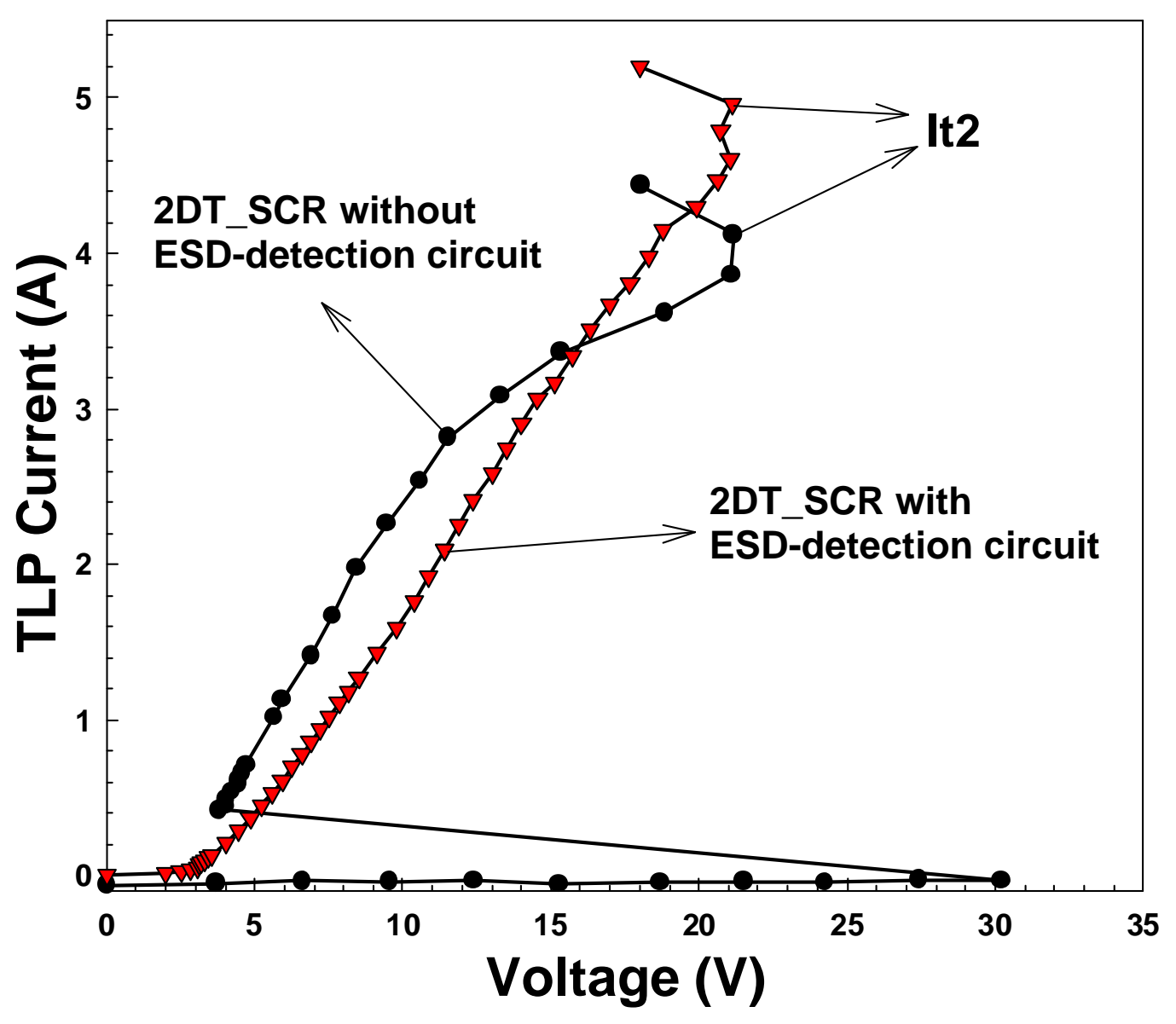

Fig. 15. The TLP-measured I-V curves of the two stacked DT_SCR devices with or without ESD-detection circuit (Failure criterion: $\mathrm{I}_{\text {Leakage }}>1 \mu \mathrm{A} @ 2.5 \mathrm{~V}$ bias). 


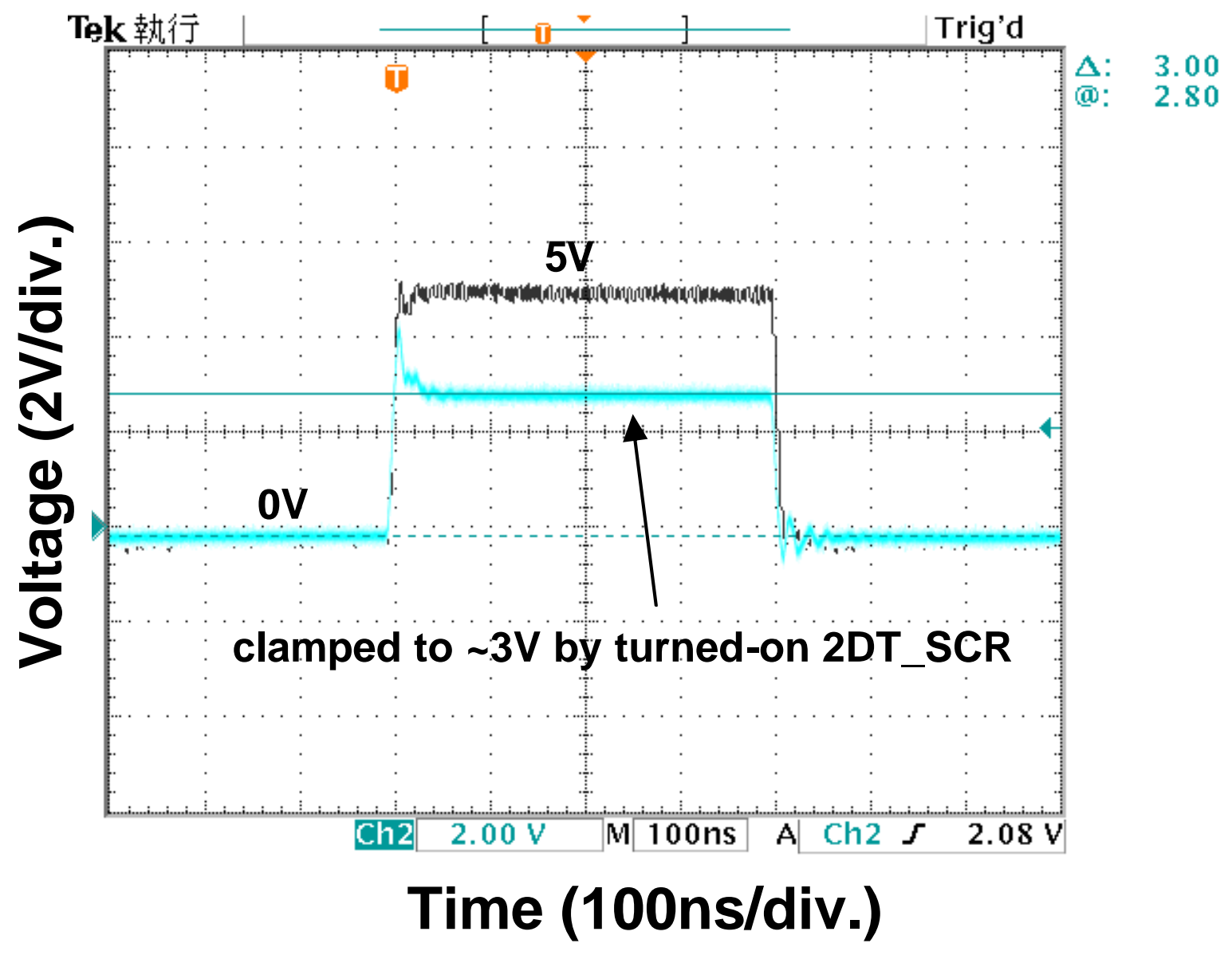

Fig. 16. The measured voltage waveforms to verify the turn-on efficiency of the power-rail ESD clamp circuit with two stacked DT_SCR devices. 